\title{
Bogoliubov Approach to Superfluidity of Atoms in an Optical Lattice
}

\author{
${ }^{3}$ Ana Maria Rey, ${ }^{1}$ Keith Burnett, ${ }^{1}$ Robert Roth, ${ }^{2}$ Mark Edwards, ${ }^{3}$ Carl J. Williams \\ ${ }^{3}$ Charles W. Clark \\ ${ }^{1}$ Department of Physics, Clarendon Laboratory \\ Parks Road, Oxford OX1 3PU, United Kingdom \\ ${ }^{2}$ Department of Physics, Georgia Southern University \\ Statesboro, GA 30460-8031, USA \\ 3 Physics Laboratory \\ National Institute of Standards and Technology \\ Technology Administration \\ U.S. Department of Commerce \\ Gaithersburg, MD 20899-8410, USA
}

October 22, 2018

\begin{abstract}
We use the Bogoliubov theory of atoms in an optical lattice to study the approach to the Mott-insulator transition. We derive an explicit expression for the superfluid density based on the rigidity of the system under phase variations. This enables us to explore the connection between the quantum depletion of the condensate and the quasi-momentum distribution on the one hand and the superfluid fraction on the other. The approach to the insulator phase may be characterized through the filling of the band by quantum depletion, which should be directly observable via the matter wave interference patterns. We complement these findings by self-consistent Hartree-Fock-Bogoliubov-Popov calculations for onedimensional lattices including the effects of a parabolic trapping potential.
\end{abstract}

\section{Introduction}

Spectacular progress has been made in experimental studies of atoms loaded into an optical lattice in the region of the Mott superfluid insulator quantum phase transition 11, 2. In this article, we shall discuss the superfluid density and the quasi-momentum distribution, which is directly related to the matterwave interference patterns that can be observed in such experiments. To do this we use the Bogoliubov method [3], as developed for use in optical lattices [4]. 
In a previous paper [5] we used this method to produce results for squeezing that are consistent with those of other approaches previously reported in the literature 6, 7, 8, 9. In this paper we shall show how it can be used to predict the decrease in the superfluid fraction and the corresponding variations in the matter wave interference fringes that should be directly observable in future experiments. This extends our previous studies based on exact calculation for small one-dimensional systems [10 into the experimentally relevant regime of lattice sizes and particle numbers.

We first introduce the Bose-Hubbard Hamiltonian for atoms in an optical lattice 11. We then describe briefly our method for determining the superfluid fraction based on the rigidity of the system under a twist of the condensate phase 12. Using a perturbative formulation analogous to the Drude weight 13 the Bogoliubov approximation gives us a particularly direct way of finding this quantity. It also gives a simple picture of how superfluidity is suppressed by quantum depletion of the condensate. We shall compare the results for various quantities calculated using the Bogoliubov approximation with exact numerical calculations for the case of modest numbers of atoms and lattice sites [10].

\section{The Bose-Hubbard Model and Superfluidity}

The Bose-Hubbard Hamiltonian for atoms in an one-dimensional optical lattice with $I$ sites has the form [1]:

$$
\hat{H}=\sum_{i=1}^{I} \hat{n}_{i} \epsilon_{i}-J \sum_{i=1}^{I}\left(\hat{a}_{i+1}^{\dagger} \hat{a}_{i}+\hat{a}_{i}^{\dagger} \hat{a}_{i+1}\right)+\frac{V}{2} \sum_{i=1}^{I} \hat{n}_{i}\left(\hat{n}_{i}-1\right)
$$

Here $J$ represents the coupling between adjacent lattice sites due to tunneling and $V$ is the strength of repulsion between atoms on the same site. The noninteracting energy of the atoms on each site, $\epsilon_{i}$, will have some variation that is typically smooth on the scale of the condensate. We shall consider below both, the case where this is a constant, as well as the extension to the case where it varies in a trapped condensate. This Bose-Hubbard Hamiltonian should be an appropriate model when the loading process produces atoms in the lowest vibrational state of each well, with a chemical potential smaller than the distance to the first vibrationally excited state. This is known to be possible from the results of recent experiments 1, 2, 14.

The concept of superfluidity is closely related to the existence of a condensate in the interacting many-body system. Formally, the one-body density matrix $\rho^{(1)}\left(\vec{x}, \vec{x}^{\prime}\right)$ has to have exactly one macroscopic eigenvalue which defines the number of particles in the condensate; the corresponding eigenvector describes the condensate wave function $\phi_{0}(\vec{x})=e^{i \Theta(\vec{x})}\left|\phi_{0}(\vec{x})\right|$. A spatially varying condensate phase, $\Theta(\vec{x})$, is associated with a velocity field for the condensate by

$$
\vec{v}_{0}(\vec{x})=\frac{\hbar}{m} \vec{\nabla} \Theta(\vec{x}) .
$$


This irrotational velocity field is identified with the velocity of the superfluid flow, $\vec{v}_{s}(\vec{x}) \equiv \vec{v}_{0}(\vec{x})([15$, , 16] $)$ and enables us to derive an expression for the superfluid fraction, $f_{s}$. Consider a system with a finite linear dimension, $L$, in the $\vec{e}_{1}$-direction and a ground-state energy, $E_{0}$, calculated with periodic boundary conditions. Now we impose a linear phase variation, $\Theta(\vec{x})=\theta x_{1} / L$ with a total twist angle $\theta$ over the length of the system in the $\vec{e}_{1}$-direction. The resulting ground-state energy, $E_{\theta}$ will depend on the phase twist. For very small twist angles, $\theta \ll \pi$, the energy difference, $E_{\theta}-E_{0}$, can be attributed to the kinetic energy, $T_{s}$, of the superflow generated by the phase gradient. Thus,

$$
E_{\theta}-E_{0}=T_{s}=\frac{1}{2} m N f_{s} \vec{v}_{s}^{2},
$$

where $m$ is the mass of a single particle and $N$ is the total number of particles so that $m N f_{s}$ is the total mass of the superfluid component. Replacing the superfluid velocity, $\vec{v}_{s}$ with the phase gradient according to Eq. (2) leads to a fundamental relation for the superfluid fraction

$$
f_{s}=\frac{2 m}{\hbar^{2}} \frac{L^{2}}{N} \frac{E_{\theta}-E_{0}}{\theta^{2}}=\frac{1}{N} \frac{E_{\theta}-E_{0}}{J(\Delta \theta)^{2}},
$$

where the second equality applies to a lattice system on which a linear phase variation has been imposed. Here the distance between sites is $a$, the phase variation over this distance is $\Delta \theta$, and the number of sites is $I$. In this case, $J \equiv \hbar^{2} /\left(2 m a^{2}\right)$.

Technically the phase variation can be imposed through so-called twisted boundary conditions [12. In the context of the discrete Bose-Hubbard model it is, however, more convenient to map the phase variation by means of a unitary transformation onto the Hamiltonian. The resulting "twisted" Hamiltonian

$$
\hat{H}_{\theta}=\sum_{i=1}^{I} \hat{n}_{i} \epsilon_{i}-J \sum_{i=1}^{I}\left(e^{-\mathrm{i} \Delta \theta} \hat{a}_{i+1}^{\dagger} \hat{a}_{i}+e^{\mathrm{i} \Delta \theta} \hat{a}_{i}^{\dagger} \hat{a}_{i+1}\right)+\frac{V}{2} \sum_{i=1}^{I} \hat{n}_{i}\left(\hat{n}_{i}-1\right)
$$

exhibits additional phase factors $e^{ \pm \mathrm{i} \Delta \theta}$ - the so-called Peierls phase factors - in the hopping term [17] 18. These phase factors show that the twist is equivalent to the imposition of an acceleration on the lattice for a finite time. It is interesting to note that the present experiments enable us to make a specific connection between the formal and operational aspects of the system.

We calculate the change in energy $E_{\theta}-E_{0}$ under the assumption that the phase change $\Delta \theta$ is small so that we can write:

$$
e^{-\mathrm{i} \Delta \theta} \simeq 1-\mathrm{i} \Delta \theta-\frac{1}{2}(\Delta \theta)^{2} .
$$

Using this expansion the twisted Hamiltonian (5) takes the following form:

$$
\hat{H}_{\theta} \simeq \hat{H}_{0}+\Delta \theta \hat{J}-\frac{1}{2}(\Delta \theta)^{2} \hat{T}=\hat{H}_{0}+\hat{H}_{\text {pert }},
$$


where we retain terms up to second order in $\Delta \theta$. The current operator $\hat{J}$ (N.B. that the physical current is given by this expression multiplied by $\frac{1}{\hbar}$ ) and the hopping operator $\hat{T}$ are given by:

$$
\begin{aligned}
& \hat{J}=\mathrm{i} J \sum_{i=1}^{I}\left(\hat{a}_{i+1}^{\dagger} \hat{a}_{i}-\hat{a}_{i}^{\dagger} \hat{a}_{i+1}\right) \\
& \hat{T}=-J \sum_{i=1}^{I}\left(\hat{a}_{i+1}^{\dagger} \hat{a}_{i}+\hat{a}_{i}^{\dagger} \hat{a}_{i+1}\right) .
\end{aligned}
$$

The change in the energy $E_{\theta}-E_{0}$ due to the imposed phase twist can now be evaluated in second order perturbation theory

$$
E_{\theta}-E_{0}=\Delta E^{(1)}+\Delta E^{(2)} .
$$

The first order contribution to the energy change is proportional to the expectation value of the hopping operator

$$
\Delta E^{(1)}=\left\langle\Psi_{0}\left|\hat{H}_{\text {pert }}\right| \Psi_{0}\right\rangle=-\frac{1}{2}(\Delta \theta)^{2}\left\langle\Psi_{0}|\hat{T}| \Psi_{0}\right\rangle .
$$

Here $\left|\Psi_{0}\right\rangle$ is the ground state of the original Bose-Hubbard Hamiltonian (1). The second order term is related to the matrix elements of the current operator involving the excited states $\left|\Psi_{\nu}\right\rangle(\nu=1,2, \ldots)$ of the original Hamiltonian

$$
\Delta E^{(2)}=-\sum_{\nu \neq 0} \frac{\left|\left\langle\Psi_{\nu}\left|\hat{H}_{\text {pert }}\right| \Psi_{0}\right\rangle\right|^{2}}{E_{\nu}-E_{0}}=-(\Delta \theta)^{2} \sum_{\nu \neq 0} \frac{\left|\left\langle\Psi_{\nu}|\hat{J}| \Psi_{0}\right\rangle\right|^{2}}{E_{\nu}-E_{0}} .
$$

Thus we obtain for the energy change up to second order in $\Delta \theta$

$$
\begin{aligned}
E_{\theta}-E_{0} & =(\Delta \theta)^{2}\left(-\frac{1}{2}\left\langle\Psi_{0}|\hat{T}| \Psi_{0}\right\rangle-\sum_{\nu \neq 0} \frac{\left|\left\langle\Psi_{\nu}|\hat{J}| \Psi_{0}\right\rangle\right|^{2}}{E_{\nu}-E_{0}}\right)=I(\Delta \theta)^{2} D \\
D & \equiv \frac{1}{I}\left(-\frac{1}{2}\left\langle\Psi_{0}|\hat{T}| \Psi_{0}\right\rangle-\sum_{\nu \neq 0} \frac{\left|\left\langle\Psi_{\nu}|\hat{J}| \Psi_{0}\right\rangle\right|^{2}}{E_{\nu}-E_{0}}\right) .
\end{aligned}
$$

The quantity $D$, defined above, is formally equivalent to the Drude weight used to specify the DC conductivity of charged fermionic systems [13. The superfluid fraction is then given by the contribution of both the first and second order term:

$$
\begin{aligned}
f_{\mathrm{s}} & =f_{\mathrm{s}}^{(1)}-f_{\mathrm{s}}^{(2)} \\
f_{\mathrm{s}}^{(1)} & \equiv-\frac{1}{2 N J}\left(\left\langle\Psi_{0}|\hat{T}| \Psi_{0}\right\rangle\right), \\
f_{\mathrm{s}}^{(2)} & \equiv \frac{1}{N J}\left(\sum_{\nu \neq 0} \frac{\left|\left\langle\Psi_{\nu}|\hat{J}| \Psi_{0}\right\rangle\right|^{2}}{E_{\nu}-E_{0}}\right) .
\end{aligned}
$$

Here $N$ is the number of atoms in the lattice. In general both, the first and the second order term contribute. For a translationally invariant lattice the second 
term vanishes (as is going to be shown latter) in the Bogoliubov limit that we shall use in this study. However, in exact calculations and in the Bogoliubov approximation for an inhomogeneous lattice the second order term plays a role.

We can further understand this approach to the superfluid density by calculating the flow that is produced by the application of the phase twist. To do this we work out the expectation value of the current operator expressed in terms of the twisted variables:

$$
\hat{J}_{\theta}=\mathrm{i} J \sum_{i=1}^{I}\left(e^{-\mathrm{i} \Delta \theta} \hat{a}_{i+1}^{\dagger} \hat{a}_{i}-e^{\mathrm{i} \Delta \theta} \hat{a}_{i}^{\dagger} \hat{a}_{i+1}\right) .
$$

We expand this to find the lowest order contributions, i.e.:

$$
\hat{J}_{\theta} \simeq \hat{J}+J \Delta \theta \sum_{i=1}^{I}\left(\hat{a}_{i+1}^{\dagger} \hat{a}_{i}+\hat{a}_{i}^{\dagger} \hat{a}_{i+1}\right)=\hat{J}-\hat{T} \Delta \theta .
$$

We use first order perturbation theory on the wave function to obtain the following expression:

$$
\begin{aligned}
\left\langle\Psi(\Delta \theta)\left|\hat{J}_{\theta}\right| \Psi(\Delta \theta)\right\rangle & =2 \Delta \theta\left(-\frac{1}{2}\left\langle\Psi_{0}|\hat{T}| \Psi_{0}\right\rangle-\sum_{\nu \neq 0} \frac{\left|\left\langle\Psi_{\nu}|\hat{J}| \Psi_{0}\right\rangle\right|^{2}}{E_{\nu}-E_{0}}\right) \\
& =2 N J f_{\mathrm{s}} \Delta \theta .
\end{aligned}
$$

If we note that the kinetic energy for a small quasi-momentum $q$ on a lattice is given by $J q^{2} a^{2}$, we can define the effective mass as $m^{*}=\frac{\hbar^{2}}{2 J a^{2}}$. Here the quasi-momenta are given by $q=\frac{2 \pi}{I a} j$ with $j=1, \ldots,(I-1)$ and lattice spacing a. Thus, the physical current, Eq. (18) multiplied by $\frac{1}{\hbar}$, can be expressed as:

$$
\left\langle\Psi(\Delta \theta)\left|\hat{J}_{\theta}\right| \Psi(\Delta \theta)\right\rangle=N f_{\mathrm{s}} \Delta \theta \frac{\hbar}{m^{*} a^{2}} .
$$

This is the total flux and we need to divide $I$ to get the flux density, i.e.

$$
\begin{aligned}
\frac{1}{I}\left\langle\Psi(\Delta \theta)\left|\hat{J}_{\theta}\right| \Psi(\Delta \theta)\right\rangle & =\left(\frac{\hbar \Delta \theta}{m^{*} a}\right)\left(\frac{N f_{\mathrm{s}}}{a I}\right) \\
& =v_{\mathrm{s}} n_{\mathrm{s}} .
\end{aligned}
$$

So we see that the Drude formulation of the superfluid fraction (14) gives an intuitively satisfying expression for the amount of flowing superfluid.

\section{The Bogoliubov Approximation to the Bose- Hubbard Hamiltonian}

We use the Bogoliubov approximation for the Bose-Hubbard model in the limit that quantum fluctuations, or equivalently depletion of the condensate, is not 
too great. In the limit that the quantum depletion can be completely ignored, we can replace the creation and annihilation operators, $\hat{a}_{i}^{\dagger}$ and $\hat{a}_{i}$, on each site with a $c$-number, $z_{i}$. This leads to a set of coupled non-linear Schrödinger, i.e. Gross-Pitaevskii (GP), equations for these amplitudes [19]:

$$
\mathrm{i} \hbar \partial_{t} z_{i}=-J\left(z_{i+1}+z_{i-1}\right)+V z_{i} z_{i}^{*} z_{i} .
$$

This equation can be used to study the properties of the condensate loaded into the lattice when the tunneling kinetic energy is large enough compared to the interaction energy though small enough for the one-band Bose-Hubbard model to be valid. We then include the quantum fluctuations in our description of the system using the Bogoliubov approximation, where we suppose that we can write the full annihilation operator in terms of the $c$-number part and a fluctuation operator thus:

$$
\hat{a}_{i}=\left(z_{i}+\hat{\delta}_{i}\right) e^{-\mathrm{i} \frac{\mu t}{\hbar}} .
$$

This form will be useful when we are looking at the properties of a timeindependent or adiabatic ground state. In using this method we are assuming that the fluctuation part is small. The Bogoliubov method gives us expressions for the averages of the squares of the fluctuation operator and allows us to determine whether this assumption is valid. We shall examine its validity by comparing the results for various physical quantities with exact numerical calculations based on the Bose-Hubbard Hamiltonian.

\subsection{Bogoliubov theory for the translationally invariant lat- tice}

The ground state solution for the translationally invariant lattice gives the eigenvalue:

$$
\mu=n_{0} V-2 J
$$

where

$$
n_{0}=N / I
$$

is the mean number of atoms on each site of the lattice. We take $N$ to be the

total number of atoms and $I$ to be the number of sites in the one-dimensional lattice.

The Bogoliubov equations for the lattice have the following form:

$$
\mathrm{i} \hbar \partial_{t} \hat{\delta}_{i}=\left(2 n_{0} V-\mu\right) \hat{\delta}_{i}-J\left(\hat{\delta}_{i+1}+\hat{\delta}_{i-1}\right)+n_{0} V \hat{\delta}_{i}^{\dagger} .
$$

This is solved by constructing quasi-particles for the lattice which diagonalize the Hamiltonian 4], i.e

$$
\begin{aligned}
& \hat{\delta}_{i}=\frac{1}{\sqrt{I}} \sum_{q}\left[u^{q} \hat{\alpha}_{q} e^{\mathrm{i}\left(q i a-\omega_{q} t\right)}-v^{q *} \hat{\alpha}_{q}^{\dagger} e^{-\mathrm{i}\left(q i a-\omega_{q} t\right)}\right] \\
& \hat{\delta}_{i}^{\dagger}=\frac{1}{\sqrt{I}} \sum_{q}\left[u^{q *} \hat{\alpha}_{q}^{\dagger} e^{-\mathrm{i}\left(q i a-\omega_{q} t\right)}-v^{q} \hat{\alpha}_{q} e^{\mathrm{i}\left(q i a-\omega_{q} t\right)}\right]
\end{aligned}
$$


where $a$ is the lattice spacing. The quasi-particle operators obey the usual Bose commutation relations:

$$
\left[\hat{\alpha}_{q}, \hat{\alpha}_{q^{\prime}}^{\dagger}\right]=\delta_{q q^{\prime}}
$$

and have the following expectation values at some temperature $T$ :

$$
\left\langle\hat{\alpha}_{q}^{\dagger} \hat{\alpha}_{q^{\prime}}\right\rangle=\delta_{q q^{\prime}}\left[\exp \left(\hbar \omega_{q} / k_{b} T\right)-1\right]^{-1} .
$$

We then find the following equations for the excitation amplitudes and frequencies,

$$
\begin{aligned}
\hbar \omega_{q} u^{q} & =\left[n_{0} V+4 J \sin ^{2}\left(\frac{q a}{2}\right)\right] u^{q}-n_{0} V v^{q}, \\
-\hbar \omega_{q} v^{q} & =\left[n_{0} V+4 J \sin ^{2}\left(\frac{q a}{2}\right)\right] v^{q}-n_{0} V u^{q} .
\end{aligned}
$$

Thus, the expressions for the $u^{q}$ and $v^{q}$ yield:

$$
\begin{aligned}
\left|u^{q}\right|^{2} & =\frac{K(q)+n_{0} V+\hbar \omega_{q}}{2 \hbar \omega_{q}} \\
\left|v^{q}\right|^{2} & =\frac{K(q)+n_{0} V-\hbar \omega_{q}}{2 \hbar \omega_{q}},
\end{aligned}
$$

where the phonon excitation frequencies are given by:

$$
\begin{aligned}
\hbar \omega_{q} & =\sqrt{K(q)\left[2 n_{0} V+K(q)\right]} \\
K(q) & =4 J \sin ^{2}\left(\frac{q a}{2}\right) .
\end{aligned}
$$

\subsection{Expressions for the number superfluid fraction in the translationally invariant lattice}

Having obtained the expressions for the excitations we can now determine the superfluid fraction. The quantity we need to calculate is just the first order term of the Drude expression (14), because the second order term vanishes in the Bogoliubov limit due to the translational invariance of the lattice [see Eq. [48]), i.e.

$$
f_{\mathrm{s}}=-\frac{1}{2 N J}\left\langle\Psi_{0}|\hat{T}| \Psi_{0}\right\rangle=\frac{1}{2 N} \sum_{i=1}^{I}\left\langle\Psi_{0}\left|\hat{a}_{i+1}^{\dagger} \hat{a}_{i}+\hat{a}_{i}^{\dagger} \hat{a}_{i+1}\right| \Psi_{0}\right\rangle .
$$

In the Bogoliubov approximation this has the form:

$$
\begin{aligned}
f_{\mathrm{s}} & =\frac{1}{2 N} \sum_{i=1}^{I}\left\langle\Psi_{0}\left|\left(\hat{\delta}_{i+1}^{\dagger}+z_{i+1}\right)\left(\hat{\delta}_{i}+z_{i}\right)+\left(\hat{\delta}_{i}^{\dagger}+z_{i}\right)\left(\hat{\delta}_{i+1}+z_{i+1}\right)\right| \Psi_{0}\right\rangle \\
& =\frac{1}{2 N} \sum_{i=1}^{I}\left\langle\Psi_{0}\left|2 z_{i}^{2}+\hat{\delta}_{i+1}^{\dagger} \hat{\delta}_{i}+\hat{\delta}_{i}^{\dagger} \hat{\delta}_{i+1}\right| \Psi_{0}\right\rangle .
\end{aligned}
$$


We can now express the fluctuation operators, Eqs. (26) and (27), in terms of the quasi-particle operators that diagonalize the quadratic Hamiltonian. This leads to the following expression for the superfluid fraction at finite temperature:

$$
\begin{aligned}
f_{\mathrm{s}}=\frac{1}{2 N}[ & \sum_{i=1}^{I} 2 z_{i}^{2}+\frac{1}{I}\left\langle\sum_{q}\left[u^{q} \hat{\alpha}_{q} e^{\mathrm{i} q(i+1) a}-v^{q} \hat{\alpha}_{q}^{\dagger} e^{-\mathrm{i} q(i+1) a}\right]\right. \\
& \left.\times \sum_{q^{\prime}}\left[u^{q^{\prime}} \hat{\alpha}_{q^{\prime}}^{\dagger} e^{-\mathrm{i} q^{\prime} i a}-v^{q^{\prime}} \hat{\alpha}_{q^{\prime}} e^{\mathrm{i} q^{\prime} i a}\right]\right\rangle \\
& +\frac{1}{I} \sum_{i=1}^{I}\left\langle\sum_{q}\left[u^{q} \hat{\alpha}_{q}^{\dagger} e^{-\mathrm{i} q i a}-v^{q} \hat{\alpha}_{q} e^{\mathrm{i} q i a}\right]\right. \\
& \left.\left.\times \sum_{q^{\prime}}\left[u^{q^{\prime}} \hat{\alpha}_{q^{\prime}} e^{\mathrm{i} q^{\prime}(i+1) a}-v^{q^{\prime}} \hat{\alpha}_{q^{\prime}}^{\dagger} e^{-\mathrm{i} q^{\prime}(i+1) a}\right]\right\rangle\right]
\end{aligned}
$$

and we find in the zero temperature limit of a translationally invariant lattice:

$$
f_{\mathrm{s}}=\frac{I}{N}\left[z^{2}+\frac{1}{I} \sum_{q}\left|v^{q}\right|^{2} \cos (q a)\right] .
$$

Here the summation runs over all quasi-momenta $q=\frac{2 \pi}{I a} j$ with $j=1, \ldots,(I-1)$ and we have called $z$ the value of all $z_{i}$ in a translationally invariant system. This shows that in the limit of zero lattice spacing (while keeping $q$ finite) the superfluid fraction is unity as we have the normalization condition:

$$
I z^{2}+\sum_{q}\left|v^{q}\right|^{2}=N .
$$

These expressions give a direct insight into the change of the superfluid fraction as atoms are pushed out of the condensate due to interactions. In Eq. (39) the sum involving the Bogoliubov amplitudes $v^{q}$ characterizes the difference between the condensate fraction, which is given by the first term, and the superfluid fraction. For weak interactions and a small depletion, which fills only the lower quarter of the band where the $\cos (q a)$ term has a positive sign, the superfluid fraction is larger than the condensate fraction. Thus the depletion of the condensate has initially little effect on superfluidity. When the depleted population spreads into the central part of the band, where the $\cos (q a)$ term has a negative sign, the superfluid fraction is reduced and might even become smaller than the condensate fraction. Finally, the population in the upper quarter of the band again produces a positive contribution to the superflow. In a sense the interactions are playing a role akin to Fermi exclusion "pressure" in the case of electron flow in a band. This, however can lead to perfect filling and cancellation of the flow. In the case of our Bogoliubov description we can only see reduction of the flow, not a perfect switching off of the superfluid. This happens in the Mott insulator state, which cannot be described by the Bogoliubov approximation. 
In the next section we outline the version of the Bogoliubov theory that should be best suited to treating these problems, i.e self-consistent Bogoliubov theory.

\section{Self-consistent HFB-Popov theory}

In this section we explore the limits of validity of the simplest zero temperature self-consistent Bogoliubov theory, a simplified version of the Hartree-FockBogoliubov approximation originally introduced by Popov [20]. The HFB-Popov theory is an extension of the standard Bogoliubov approximation that takes into account the depletion of the condensate but neglects the anomalous average. As discussed in the previous section, taking into account the depletion of the condensate is important as the transition is approached because the depleted population causes the reduction of the superfluidity. Although the HFB-Popov aproach has the limitation that it doesn't take into account the full effect of the medium because it neglects the anomalous average [21, it can be considered a better theory for the elementary excitations than the full HFB due to the fact that the theory is gapless and doesn't violate Goldstone's theorem.

A derivation of the Bogoliubov equations for the quasiparticle amplitudes in a lattice can be found for example in Ref. [5]. These equations only take into account terms up to second order in the fluctuations. Including third and fourth order terms by treating them in a self-consistent mean field approximation ( [22], [23] ) and neglecting anomalous average terms yields the following HFB-Popov equations:

$$
\begin{aligned}
\hbar \omega_{q} u_{i}^{q}+c^{q} z_{i} & \left.=\left(2 V\left(\left|z_{i}\right|^{2}+\tilde{n}_{i}\right)-\mu+\epsilon_{i}\right) u_{i}^{q}-J\left(u_{i+1}^{q}+u_{i-1}^{q}\right)-V z_{i}^{2} v_{\hat{\alpha}}^{q} 41\right) \\
-\hbar \omega_{q} v_{i}^{q}-c^{q} z_{i}^{*} & \left.=\left(2 V\left(\left|z_{i}\right|^{2}+\tilde{n}_{i}\right)-\mu+\epsilon_{i}\right) v_{i}^{q}-J\left(v_{i+1}^{q}+v_{i-1}^{q}\right)-V z_{i}^{* 2} u_{i}^{q} 42\right) \\
\mu z_{i} & =-J\left(z_{i+1}+z_{i-1}\right)+\left(V\left(\left|z_{i}\right|^{2}+2 \tilde{n}_{i}\right)+\epsilon_{i}\right) z_{i} \\
\tilde{n}_{i} & =\sum_{q}\left|v_{i}^{q}\right|^{2} \\
N & =\sum_{i=1}^{I}\left(\left|z_{i}\right|^{2}+\tilde{n}_{i}\right) \\
c^{q} & =V \sum_{i}\left|z_{i}\right|^{2}\left(z_{i}^{*} u_{i}^{q}-z_{i} v_{i}^{q}\right)
\end{aligned}
$$

Where $\epsilon_{i}$ is the energy offset at site $i$ due to an external potential, $\left\{u_{i}^{q}, v_{i}^{q}\right\}$ and $\omega_{q}$ are respectively the quasiparticle amplitudes and energies, thus

$$
\hat{\delta}_{i}=\sum_{q} u_{i}^{q} \hat{\alpha}_{q} e^{-\mathrm{i} \omega_{q} t}-v_{i}^{q *} \hat{\alpha}_{q}^{\dagger} e^{\mathrm{i} \omega_{q} t}
$$

$\tilde{n}_{i}$ is the depletion at site $i$, and $N$ is the total number of particles. The parameters $c^{q}$ ensure the $\left\{u_{i}^{q}, v_{i}^{q}\right\}$ solutions to the above equations with $\omega_{q} \neq 0$ to be orthogonal to the condensate (Ref. [23]). 
By calculating the quasiparticle amplitudes and the condensate density it is possible to get information about most of the physical properties of the system. For example the superfluid fraction and the on site number fluctuations are given by:

$$
\begin{aligned}
& f_{\mathrm{s}}=f_{s}^{(1)}-f_{s}^{(2)} \\
& f_{s}^{(1)}=\sum_{i=1}^{I} f_{s i}^{(1)}=\frac{1}{2 N} \sum_{i=1}^{I}\left[\left(z_{i+1} z_{i}^{*}+z_{i+1}^{*} z_{i}\right)+\sum_{q}\left(v_{i}^{q} v_{i+1}^{q *}+v_{i}^{q *} v_{i+1}^{q}\right)\right] \\
& f_{s}^{(2)}= \frac{J}{N}\left(\sum_{q, q^{\prime}} \frac{\left|\sum_{i}\left(u_{i+1}^{q} v_{i}^{q^{\prime}}-u_{i}^{q} v_{i+1}^{q^{\prime}}\right)\right|^{2}}{\hbar \omega_{q}+\hbar \omega_{q^{\prime}}}+\delta_{q q^{\prime}} \frac{\left|\sum_{i}\left(u_{i+1}^{q} v_{i}^{q}-u_{i}^{q} v_{i+1}^{q}\right)\right|^{2}}{2 \hbar \omega_{q}}\right) \\
& \Delta n_{i}^{2}=\left|z_{i}\right|^{2} \sum_{q}\left|u_{i}^{q}-v_{i}^{q *}\right|^{2}
\end{aligned}
$$

From the complete expression of the superfluid fraction, it can be seen explicitly how due to the translational invariance, the second order term vanishes in the homogeneous system.

\subsection{Translationally Invariant lattice}

For the translational invariant lattice we use the quasiparticle transformation given by Eqs. (26) and (27). Under this transformation the self consistent equations can be written, generalizing the previous version, as:

$$
\begin{aligned}
\mu & =\left(|z|^{2}+\frac{2}{I} \sum_{q}\left|v^{q}\right|^{2}\right) V-2 J \\
\left|u^{q}\right|^{2} & =\frac{K(q)+|z|^{2} V+\hbar \omega_{q}}{2 \hbar \omega_{q}} \\
\left|v^{q}\right|^{2} & =\frac{K(q)+|z|^{2} V-\hbar \omega_{q}}{2 \hbar \omega_{q}} \\
N & =I|z|^{2}+\sum_{q}\left|v^{q}\right|^{2}
\end{aligned}
$$

Here the phonon excitation spectrum is given by:

$$
\hbar \omega_{q}=\sqrt{K(q)\left[2|z|^{2} V+K(q)\right]},
$$

and $K(q)$ is given by Eq. (35). Again we omit the subscript in the amplitudes $z_{i}$ because they have the same value at all lattice sites. Notice also that due to translational invariance, the $c^{q}$ coefficients vanish.

In the homogeneous system, the form of the HFB-Popov equations for the quasiparticle amplitudes and energies is very close to the standard Bogoliubov form. We do, however, have to replace $n_{0}=N / I$ by the condensate amplitude $|z|^{2}$ which must take into account the depletion of the condensate. We solve 
for the condensate amplitude as a function of the external parameters $J, V, N$ and $I$ by inserting Eq. (52) in Eq. (53). Once $|z|^{2}$ is determined, we use it to calculate the other expressions.

In Fig. 1 we compare the number fluctuations on a lattice site, the condensate fraction and the total and second order superfluid fraction determined from the exact solution of the Bose-Hubbard Hamiltonian to the self consistent HFB-Popov predictions as a function of the ratio $V_{\text {eff }}=V / J$. The systems used for the comparisons have three wells, $I=3$, and commensurate filling factors $n_{0}=5,10,20$ and 50. Similar results for the incommensurate case with $N=16,31,61,151$ are shown in Fig. 22 We were restricted to consider only three wells due to computational limitations. The size of the matrix needed in the exact solution for $N$ atoms and $I$ wells scales as $\frac{(N+I-1) !}{N !(I-1) !}$. However, if the HFB-Popov approach works well for these small systems we expect it to provide a good description of the larger systems prepared in the lab.

Because the second order term of the superfluid fraction (second term of Eq 14) vanishes in the HFB-Popov approach (see Eq. 48), we only expect the self consistent HFB-Popov theory to give a good description of the superfluid fraction in the region where the second order term is extremely small, provided it predicts accurately the first order term. This is exactly what is observed in the

plots. When the second order term starts to grow, typically above $0.5 V_{\text {eff }}^{\text {crit }}$, the HFB-Popov theory starts to fail. An estimate of $V_{e f f}^{\text {crit }}$ is shown by a vertical line in some of the figures. This was obtained by using the second order perturbative approach presented in Ref. 4. With increasing filling factor the critical value is shifted towards larger values of the interaction strength, and the region in which the HFB-Popov theory is accurate gets larger. It is interesting to note that the number fluctuations predicted by the theory are accurate in a greater range than the other physical quantities shown. Its predictions of squeezing agree very well with the exact solutions right up to the point where the number fluctuations become less than unity.

For the cases with non-commensurate fillings depicted in Fig. 2] the agreement is significantly better for all quantities. This is not surprising because when the filling is not commensurate there is always a superfluid present and the Mott transition doesn't occur. As can be seen in the plots for these cases the second order term is always very small.

\subsection{Inhomogeneous lattice}

In this section we consider the experimentally relevant case when there is an external magnetic confinement in addition to the lattice potential. In this situ- 

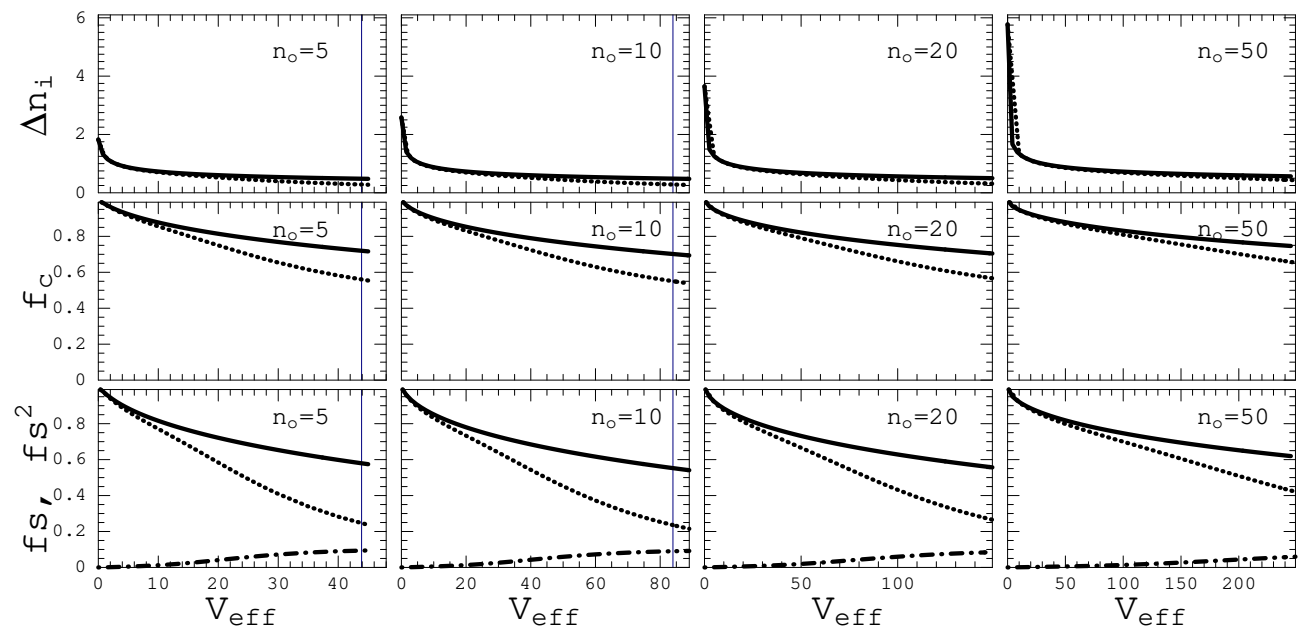

Figure 1: Comparisons of the exact solution (dotted line) and HFB-Popov (solid line) as a function of $V_{\text {eff }}=V / J$, for a system with $I=3$ and filling factors $n_{o}=5,10,20$, and 50. Top: number fluctuations, middle: condensate fraction, bottom: superfluid fraction $f_{\mathrm{s}}$. The exact second order term (dashed line) of the superfluid fraction, $f_{\mathrm{s}}^{(2)}$ is also shown in these plots. The vertical line shown in some plots is an estimation of $V_{e f f}^{c r i t}$.

ation, the self consistent HFB-Popov equations take the form:

$$
\begin{aligned}
\hbar \omega_{q} u_{i}^{q}+c^{q} z_{i} & =\left(2 V\left(\left|z_{i}\right|^{2}+\tilde{n}_{i}\right)-\mu+\Omega i^{2}\right) u_{i}^{q}-J\left(u_{i+1}^{q}+u_{i-1}^{q}\right)-V z_{i}^{2} v_{i}^{q}(55) \\
-\hbar \omega_{q} v_{i}^{q}-c^{q} z_{i}^{*} & \left.=\left(2 V\left(\left|z_{i}\right|^{2}+\tilde{n}_{i}\right)-\mu+\Omega i^{2}\right) v_{i}^{q}-J\left(v_{i+1}^{q}+v_{i-1}^{q}\right)-V z_{i}^{* 2} u u_{i}^{q} 56\right) \\
\mu z_{i} & =-J\left(z_{i+1}+z_{i-1}\right)+\left(V\left(\left|z_{i}\right|^{2}+2 \tilde{n}_{i}\right)+\Omega i^{2}\right) z_{i} \\
\tilde{n}_{i} & =\sum_{q}\left|v_{i}^{q}\right|^{2} \\
N & =\sum_{i}\left(\left|z_{i}\right|^{2}+\tilde{n}_{i}\right) \\
c^{q} & =V \sum_{i}\left|z_{i}\right|^{2}\left(z_{i}^{*} u_{i}^{q}-z_{i} v_{i}^{q}\right)
\end{aligned}
$$

where $\Omega=\frac{1}{2} m \omega^{2} a^{2}$, with $m$ the mass of the atoms, $\omega$ the trap frequency, and $a$ the lattice spacing. The site indices $i$ are chosen such that $i=0$ corresponds to the center of the trap. Again the $c^{q}$ are introduced to ensure the orthogonality of the excitations to the condensate 23. We have solved the HFB-Popov equations 

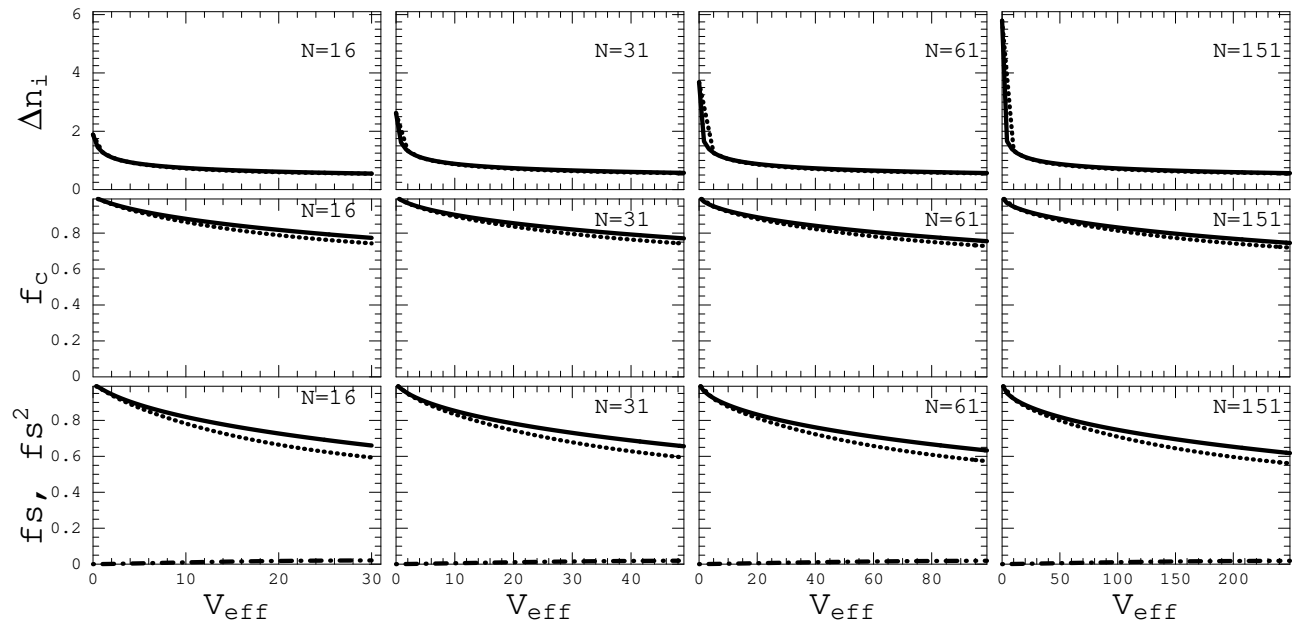

Figure 2: Comparisons of the exact solution (dotted line) and HFB-Popov (solid line) for a system with $I=3$ and non commensurate filling factors $N=16,31,61,151$ as a function of $V_{e f f}=V / J$. Top: number fluctuations, middle: condensate fraction, bottom: superfluid fraction $f_{\mathrm{s}}$. In these plots the exact second order term of the superfluid fraction is also shown with a dashed line.

for this system by an iterative procedure, similar to the one followed in Ref. 24. Each cycle of the iteration consists of two steps. In the first step we solve Eq. (57) subject to the constraint Eq. (59) by using the $\tilde{n}_{i}$ obtained in the previous cycle. This generates new values for the $z_{i}$. In the second step we solve for $\left\{u_{i}^{q}, v_{i}^{q}\right\}$ in Eqs. (55) using the $\tilde{n}_{i}$ from the previous cycle and the newly generated $z_{i}$. The $\left\{u_{i}^{q}, v_{i}^{q}\right\}$ are used then to update $\tilde{n}_{i}$. Because the HFBPopov is gapless, it is possible to keep the orthogonality of the excitations to the condensate by solving Eqs. [55] with the $c^{q}$ set to zero but removing in each cycle the projection of the calculated $\left\{u_{i}^{q}, v_{i}^{q}\right\}$ amplitudes onto the condensate. Convergence is reached when the change in $\sum_{i}\left|\tilde{n}_{i}\right|^{2}$ from one cycle to the next is smaller than a specified tolerance.

The parameters chosen for the numerical calculations were $\Omega=0.0015 E_{R}$, with $E_{R}$ the one photon recoil energy, which for the case of a Rubidium condensate corresponds to a trap frequency of approximately $90 \mathrm{~Hz}$. We used a total number of 1000 atoms, $N=1000$, and set $V N=1.0 E_{R} . J$ was varied to achieve a range of $V_{\text {eff }}=V / J$ between 0.01 and 312 . The range was chosen based on a local mean field approach [4, which for our parameters estimates 
the transition region between $V_{\text {eff }} \approx 640$ (at the center where the local filling factor is approximately 80 ) and $V_{\text {eff }} \approx 12$ (at the wings).

The results of the numerical calculations are summarized in Figs. 3 to 8 In Fig. 3 we plot the evolution of the density profile (black boxes), the condensate population (triangles) and the on-site depletion (empty diamonds) as $V_{e f f}$ is increased. In the plots we also show, for comparison purposes, the ground state density profile for $J=0$ (empty boxes). This has the advantage that can be calculated exactly from the Hamiltonian. In general we observe the reduction of the condensate population and thus the increment of the depletion as the interaction strength is increased. When the system is in the superfluid regime most of the atoms are in the condensate but as $J$ is decreased the depletion of the condensate becomes very important.

For the chosen parameters, the density profile has a parabolic shape reflecting the confining potential. By comparing the evolution of the density as $J$ is decreased with the exact solution at $J=0$, we can crudely estimate the validity of the HFB-Popov calculations. The density evolves from a Gaussian type (see plots for $V_{e f f}=0.01$ and 0.09 ) with smooth edges towards a Thomas-Fermi profile with sharp edges adjusting its shape to the $J=0$ profile. We can appreciate that around $V_{\text {eff }}=3$ both profiles are almost equal. For lower values of $J$ the HFB-Popov density starts to differ from the $J=0$ one, even though the system is closer to the $J=0$ limit. We can say that beyond this point higher order correlations, neglected by the theory, begin to be important. The departure of the HFB-Popov density profile from the $J=0$ one as $J$ is decreased begins at the edges (see panel corresponding to $V_{\text {eff }}=11$ and 100). This is something expected if we look at the on-site depletion. For such values of $V_{\text {eff }}$ the local depletion at the wings corresponds to a considerable percentage of the condensate populations, and thus the validity of the HFBPopov assumptions starts to be dubious. The homogeneous results shown in the previous section corroborate our present statements for the confined system. For the smallest filling factor (see Fig (1) the differences between the homogeneous HFB-Popov calculations and the exact solutions become important for values of $V_{\text {eff }}$ greater than 20. For higher values of $V_{\text {eff }}$, see plot for $V_{\text {eff }}=312$, the HFB-Popov density predictions differs from the $J=0$ solution even at the central wells. At this point the failure of the method is clear and a fully quantal method is required.

The HFB-Popov quasiparticle spectrum is shown in Fig 4 It can be observed how the lower energy eigenvalues evolve from a linear non degenerated spectrum to an almost degenerated one as $\mathrm{J}$ is decreased. It is worth it to mention that the small energy difference between the ground and first excited states for high values of $V_{\text {eff }}$ makes the numerical solution very unstable in the sense that it was very easy to jump to an excited state when solving for the condensate wave function. The decrement in the energy spacing predicted by the HFB-Popov theory as the system approaches the transition is very useful to keep in mind 
for the experimental realization of the Mott transition. As the optical lattice depth is ramped up the adiabaticity criteria is harder to fulfill.

In Fig. 5 we plot the results for the number fluctuations found numerically using the inhomogeneous HFB-Popov approach. The number fluctuations profile reflects the condensate profile. We also show the number fluctuations evaluated by using a local density approximation (empty boxes). The latter was calculated by substituting in the number fluctuations expression (Eq. 49) the $\left\{u^{q}, v^{q}\right\}$ amplitudes found for the homogeneous system (Eqs. 51] and [52), but replacing the condensate density in each lattice site by the one found numerically for the trapped system (see Fig. 3). The complete agreement between the two approaches justifies the validity of the local density approximation for the estimations of local quantities in confined systems. Based on this agreement and the results for the homogeneous system shown in the previous section, we expect that the inhomogeneous HFB-Popov results for squeezing also agrees with the exact solution right up to the transition.

\subsection{Quasi-momentum distribution in the inhomogeneous lattice}

The quasi-momentum distribution of the atoms released from the lattice is important because it is one of the most easily accessible quantities to the experiments. The quasi-momentum distribution function $n_{q}$ is defined as [10]

$$
n_{q}=\sum_{i, j} e^{\mathrm{i} q(i-j) a}\left\langle a_{i}^{\dagger} a_{j}\right\rangle,
$$

where the quasi-momentum $q$ can assume discrete values which are integer multiples of $\frac{2 \pi}{I a}, a$ is the lattice spacing. In Fig. [6 we present the quasi-momentum distribution for the same parameters used in the previous section. The distribution for the two lowest values of $V_{\text {eff }}$ corresponds to the one that characterizes an uncorrelated superfluid phase with a narrow peak at small quasi-momenta. As the hopping rate is decreased we observe that the sharpness of the central peak decreases and the distribution extends towards large quasi-momenta. It is interesting to note the appearance of a small peak between $q=0.5$ and 1 which is most noticeable for the $V_{\text {eff }}=3$ case. This agrees with the results found in [25] where they solve numerically the Bose-Hubbard Hamiltonian by using Monte Carlo simulations. We attribute the origin of the small peak to the depletion of the condensate at the wings. For the parameters when the small peak is present, the most important contribution to the quasi-momentum distribution still comes from the condensate atoms. The step function like shape of the condensate profile causes an oscillatory $|\sin (x) / x|$ shape of the quasi-momentum distribution. As the lattice depth is increased the hopping becomes energetically costly, the long-range order starts to decrease and the Fourier spectrum becomes broader. 
In Fig. 7 we plot the first order on site superfluid fraction $f_{s i}^{(1)}$ which was defined in Eq. (48). The curves corresponding to $V_{\text {eff }}=0.01-11$, which are in the regime where the HFB-Popov is expected to be valid, depict how as $V_{\text {eff }}$ is increased the superfluid profile decreases faster at the wings and at the center but no major change is observed in the middle section. The evolution of the on-site superfluidity as the interaction strength is increased, exhibiting a domain localized decrement instead of a global one, is in agreement with the development of uncompressible regions surrounded by superfluid rings predicted for trapped systems [26] as the transition is approached.

Fig. 8 shows the first order and total superfluid fraction and also the second order superfluid fraction as a function of $V_{\text {eff }}$. Different from the translationally invariant case, the second order contribution calculated in the HFB-Popov approach doesn't vanish for the inhomogeneous system. The rapid decrement of the superfluid fraction observed after $\log \left(V_{\text {eff }}\right) \sim 1.2$ is a signature that the system is entering a highly correlated regime. Beyond this point higher order correlations neglected in the HFB-Popov approach become crucial and a more accurate approach is required.

The Mott transition is a quantum phase transition and as all critical phenomena its behavior depends strongly on the dimensionality of the system. In the present analysis, due to computational limitations, we considered one dimensional systems. Experimentally, the Mott transition has been achieved [2] in a 3 dimensional lattice with filling factors between 1 and 3. Even though the HFB-Popov approach fails to describe the strong coupling regime for the one dimensional systems we considered in the present paper, we showed how the method is incredibly powerful in describing most of its characteristic features as they are driven from the superfluid regime towards the transition. We expect the HFB-Popov method to give a better description of the transition as the dimensionality of the system is increased and therefore to be a good model in an experimental situation.

As shown in previous studies [27, 28] the Mott transition in a d-dimensional homogeneous system has two different critical behaviors: one $(\mathrm{d}+1) \mathrm{XY}$ - like, for systems with fixed integer density as the interaction strength is changed, and one mean field-like exhibited when the transition is induced by changing the density. Different from the homogeneous case where the Mott transition is characterized by the global offset of the superfluidity, for confined systems, commensuration is only well defined locally. The inhomogeneity introduced by the confined potential allows the existence of extended Mott domains (above a critical interaction strength) surrounded by superfluid ones [26, thus the total superfluid fraction doesn't vanishes in the Mott regime. This issue, together with the fact that the finite length scale introduced by the trap suppresses the long wave fluctuations which are responsible for destroying the mean field $[23]^{1}$, make us believe the critical behavior in confined systems to be more mean-field like. Because the critical dimension for the latter type of transition is two [27],

\footnotetext{
${ }^{1}$ One obvious consequence of this is that BEC is possible in one and two dimensions in a trap whereas in the homogeneous, thermodynamic limit it can not occur in fewer than three dimensions
} 
28], we expect that for trapped systems in $d=3$, the range of validity of the HFB-Popov extends closer to the transition.

\section{Summary}

We have developed in this article a Bogoliubov method for describing the approach of a condensate loaded in an optical lattice towards the Mott transition. We have shown that this method can be used to predict the relevant physical quantities over a useful range. We have also shown how it gives a powerful insight into the way quantum depletion reduces the long range order and the superfluid fraction.

\section{Acknowledgments}

This work was supported in part by US National Science Foundation grants PHY-0100634 and PHY-0100767, the United Kingdom's Engineering and Physical Sciences Research Council, the Cold Quantum Gases Network Research Training Network, and the Advanced Research and Development activity.

\section{References}

[1] C. Orzel, A. K. Tuchman, M. L. Fenselau, M. Yasuda and M. A. Kasevich "Squeezed states in a Bose-Einstein Condensate" Science 291, 2386 (2001)

[2] M. Greiner, O. Mandel, T. Esslinger, T. W. Hänsch, and I. Bloch, "Quantum phase transition from a superfluid to a Mott insulator in a gas of ultracold atoms," Nature 415, 39 (2002)

[3] N. N. Bogoliubov, "On the theory of superfluidity" J. Phys. (USSR) 11, $23(1947)$

[4] D. van Oosten, P. van der Straten, H. T. C. Stoof, "Quantum phases in an optical lattice" Phys. Rev. A 63, 053601 (2001)

[5] K. Burnett, M. Edwards, C. W. Clark and M. Shotter, "The Bogoliubov approach to number squeezing in an optical lattice", J. Phys. B: At. Mol. Opt. Phys. 35, 1671 (2002).

[6] J. Javanainen and M. Yu. Ivanov, "Splitting a trap containing a BoseEinstein condensate: atom number fluctuations," Phys. Rev. A 60, 2351(1999)

[7] J. Javanainen, "Phonon approach to an array of traps containing BoseEinstein condensates," Phys. Rev. A 60, 4902 (1999)

[8] M. Shotter, " A Gutzwiller approach to squeezed states in an optical lattice," J. Phys. B: At. Mol. Opt. Phys. 35, 3019-3028 (2002) 
[9] G. Baym, "Topics in the microscopic theory of Bose-Einstein condensates," J. Phys. B: At. Mol. Opt. Phys. 34, 4541-4550 (2001)

[10] R. Roth, K. Burnett, "Superfluidity and interference pattern of ultracold bosons in optical lattices", arXiv:cond-mat/0209066 (2002)

[11] D. Jaksch, C. Bruder, C. W. Gardiner, J.I. Cirac and P. Zoller, "Cold bosonic atoms in optical lattices," Phys. Rev. Lett. 81, 3108 (1998)

[12] M. E. Fisher, M. N. Barber, and D. Jasnow, "Helicity modulus, superfluidity, and scaling in isotropic systems," Phys. Rev. A 8, 1111 (1973)

[13] B. S. Shastry and B. Sutherland, "Insulator, metal or superconductor: The criteria," Phys. Rev. B 47, 7995 (1993)

[14] S. Rolston et al (private communication)

[15] A. J. Legett, "Superfluidity," Rev. Mod. Phys. 71, S318 (1999)

[16] E. M. Lifshitz and L. Pitaevskii, Statistical Physics part 2, Pergamon (Oxford, 1980)

[17] D. Poilblanc, "Twisted boundary conditions in cluster calculations of the optical conductivity in two-dimensional lattice models," Phys. Rev. B 44, $9562(1991)$

[18] B. S. Shastry and B. Sutherland, "Twisted boundary conditions and effective mass in Heisenberg-Ising and Hubbard rings," Phys. Rev. Lett. 65, $243(1990)$

[19] L. Amico and V. Penna, "Time dependent mean-field theory of superfluidinsulator phase transition," Phys. Rev. B 62, 1224 (2000)

[20] V. N. Popov, Functional Integrals and Collective Modes, (Cambridge University Press, New York 1987)

[21] N.P. Proukakis, S.A. Morgan, S. Choi and K. Burnett, "Comparison of gapless mean-field theories for trapped Bose-Einstein condensates, " Phys. Rev. A 58, 2435 (1998)

[22] A. Griffin, "Conserving and gapless approximation for an inhomogeneous approximation Bose gas at finite temperature," Phys. Rev. B, 53, 9341 (1995).

[23] S. A. Morgan, A gapless theory of Bose-Einstein Condensation in Dilute Gases at Finite Temperature, D. Phil. Thesis, Oxford University (unpublished, 1999)

[24] R. J. Dodd, M. Edwards, C. W. Clark and K. Burnett, "Collective excitations of Bose-Einstein-condensed gases at finite temperature," Phys. Rev. A, 57, R32 (1998). 
[25] V. A. Kashurnikov, N. V. Prokof'ev and B. V. Svistunov, "Revealing superfluid-Mott-insulator transition in an optical lattice," arXiv:cond-mat/0202510 (2002).

[26] G. G. Batrouni et al.," Mott domains of bosons cofined on optical lattices", Phys. Rev. Lett, 89, 117203 (2002).

[27] M. P. A. Fisher, P. B. Weichman, G. Grinstein and D. S. Fisher, "Boson localization and the superfluid-insulator transition," Phys. Rev. B,40, 546 (1989).

[28] G. G. Batrouni, R. T. Scalettar and G. T. Zymanyi, "Quantum critical phenomena in one-dimensional Bose systems," Phys. Rev. Lett. 65, 1765 (1990). 


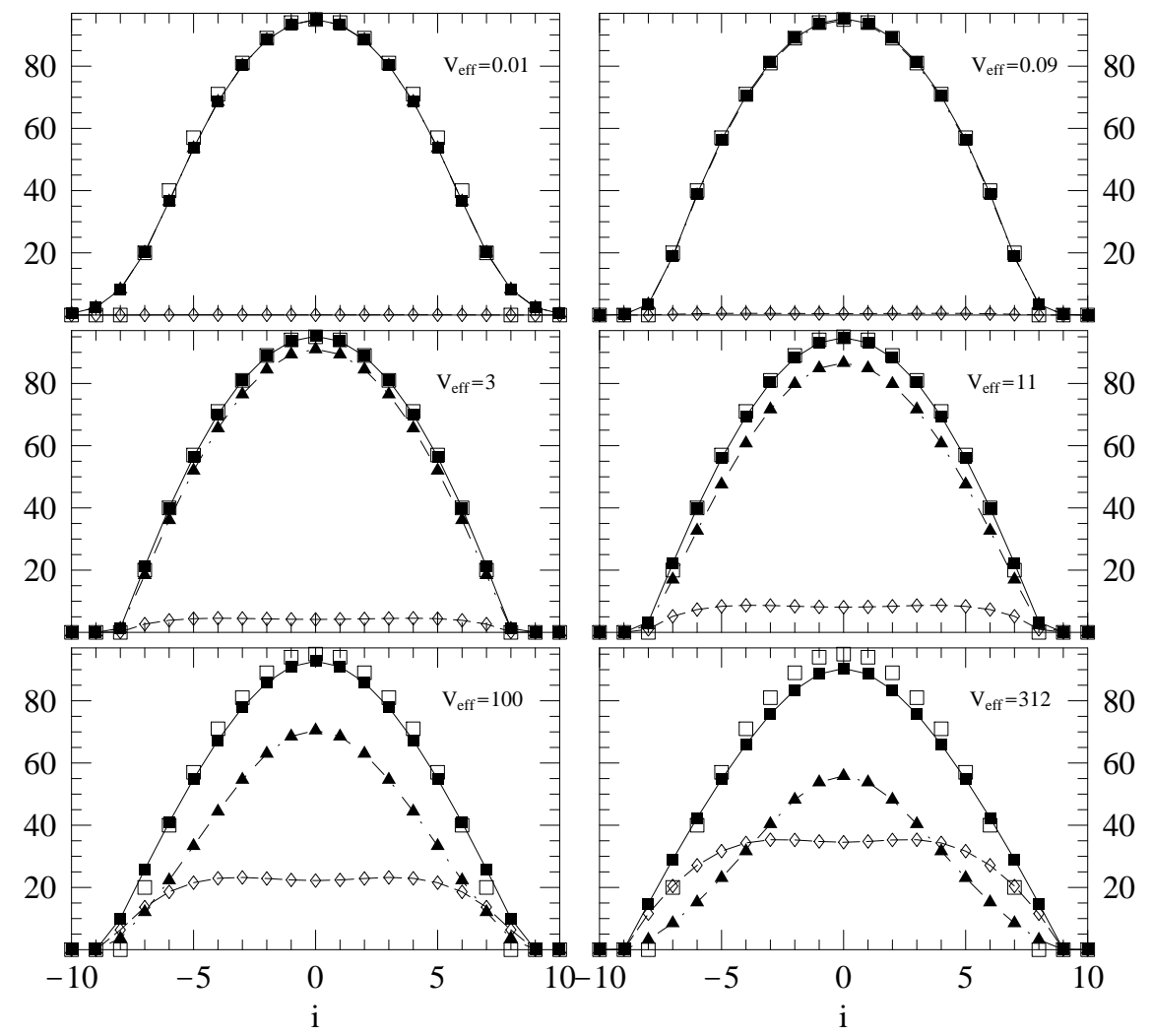

Figure 3: Condensate density (triangles), total density (filled boxes) and local depletion (empty diamonds) as a function of the lattice site for different values of $V_{\text {eff. }}$. Although these quantities are defined only at the discrete lattice sites we join them to help visualization. The empty boxes represent the exact solution for the case $\mathrm{J}=0$. 


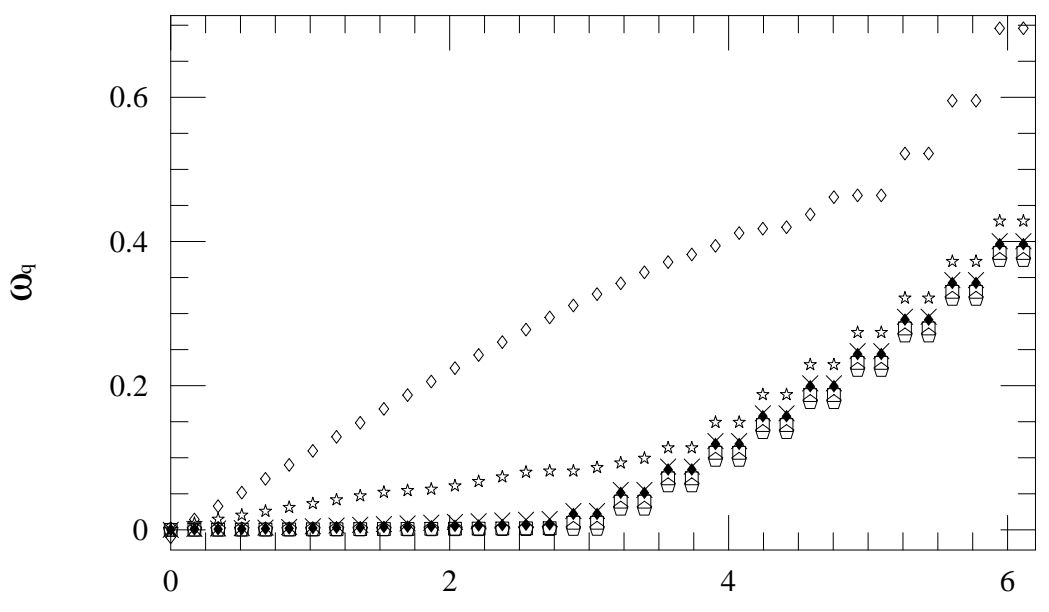

q

Figure 4: Quasiparticle spectrum in ascending order predicted by the HFBPopov theory for different values of $V_{\text {eff }}$ : Empty diamonds $\left(V_{\text {eff }}=0.01\right)$, stars $\left(V_{\text {eff }}=0.09\right)$, crosses $\left(V_{\text {eff }}=3\right)$, filled diamonds $\left(V_{\text {eff }}=11\right)$, empty boxes $\left(V_{e f f}=100\right)$ and polygons $\left(V_{\text {eff }}=312\right)$ 


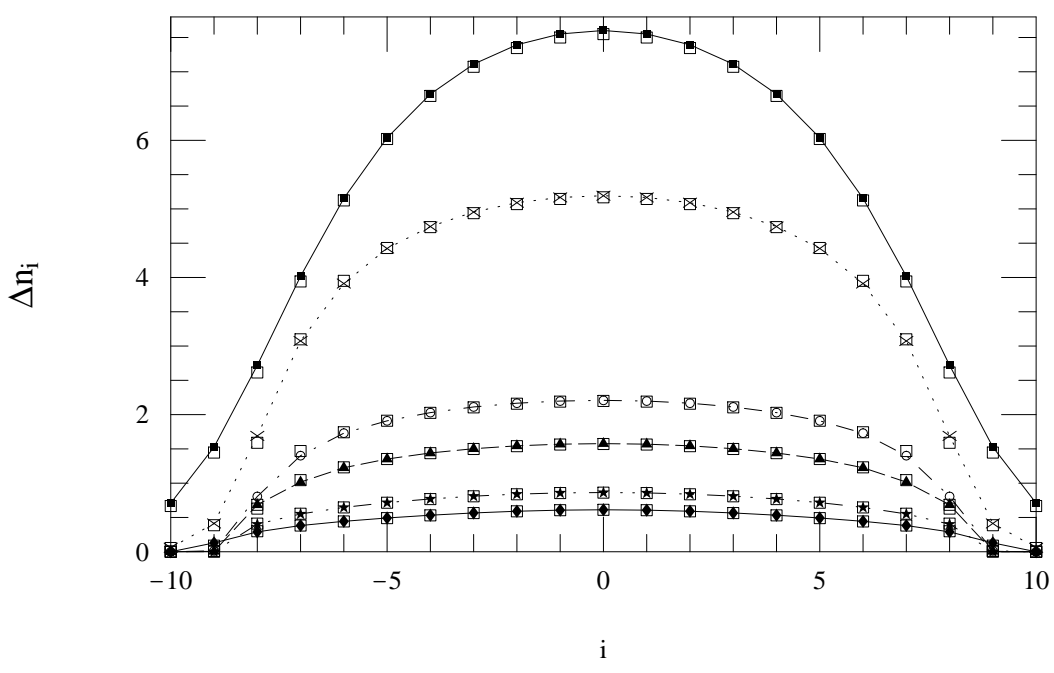

Figure 5: Number fluctuations in the self consistent HFB-Popov approach as a function of lattice site for $V_{e f f}=0.01$ (boxes), $V_{\text {eff }}=0.09$ (crosses), $V_{\text {eff }}=3$ (circles), $V_{e f f}=11$ (triangles), $V_{e f f}=100$ (stars) and $V_{e f f}=312$ (diamonds). The maximum value reached by the profile decreases as $V_{\text {eff }}$ is increased. The empty boxes shown for each of the curves correspond to the number fluctuations predicted by the homogeneous HFB-Popov model using a local density approximation. 


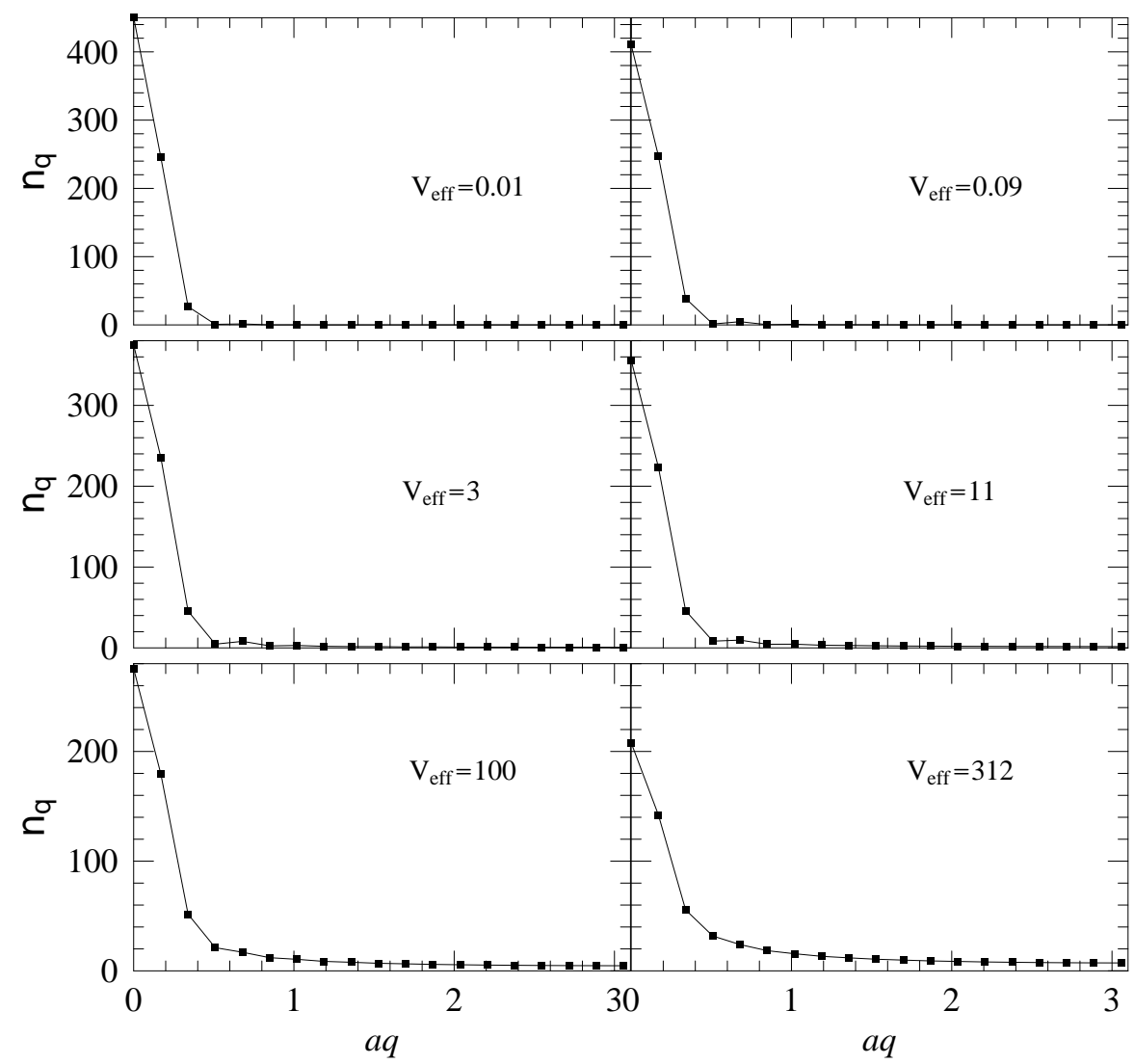

Figure 6: Quasi-momentum distribution as a function of $q a, a$ the lattice spacing, $q$ the quasimomentum, for different values of $V_{\text {eff }}$. 


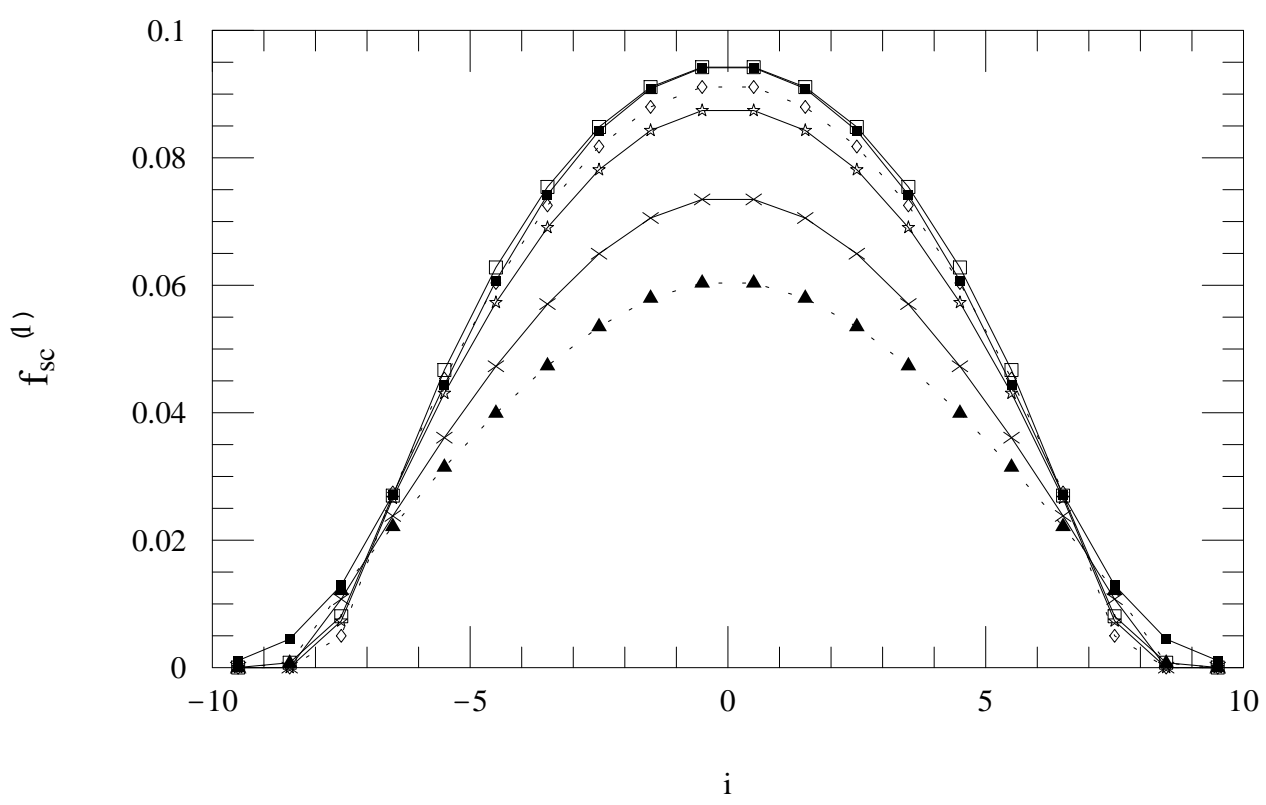

Figure 7: First order on-site superfluid fraction as a function of the lattice site for different values of $V_{\text {eff }}$. Filled boxes: $V_{\text {eff }} .=0.01$, empty boxes: $V_{\text {eff. }}=0.09$, empty diamonds: $V_{\text {eff. }}=3$, stars: $V_{\text {eff. }}=11$, crosses: $V_{\text {eff. }}=100$ and triangles: $V_{\text {eff. }}=312$. 

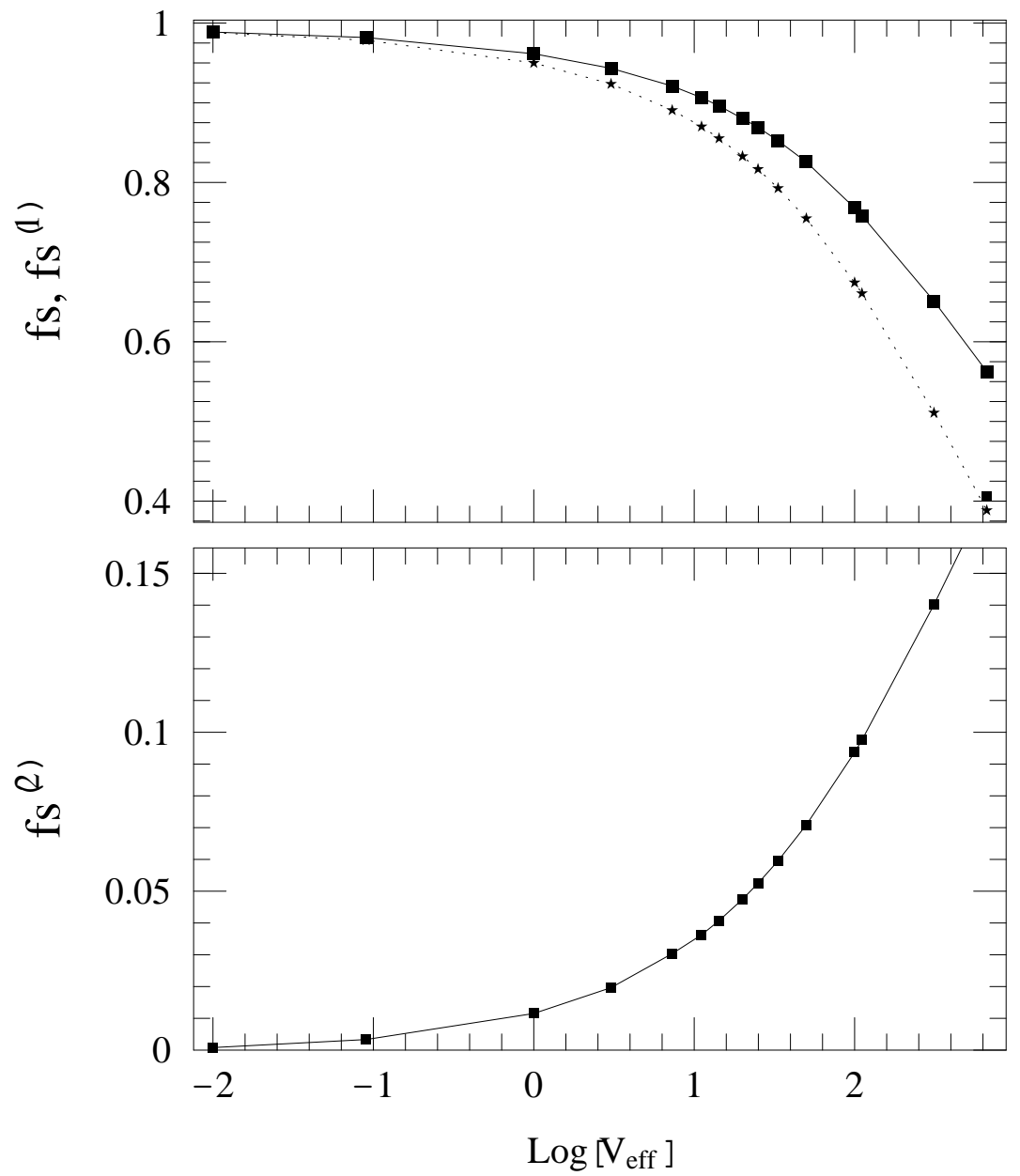

Figure 8: Top panel: First order (boxes) and total (stars) superfluid fraction as a function of $V_{\text {eff. }}$. Bottom panel : second order superfluid fraction as a function of $V_{e f f}$. All these quantities are calculated in the self consistent HFBPopov approach. 


\title{
Bogoliubov Approach to Superfluidity of Atoms in an Optical Lattice
}

\author{
${ }^{3}$ Ana Maria Rey, ${ }^{1}$ Keith Burnett, ${ }^{1}$ Robert Roth, ${ }^{2}$ Mark Edwards, ${ }^{3}$ Carl J. Williams \\ ${ }^{3}$ Charles W. Clark \\ ${ }^{1}$ Department of Physics, Clarendon Laboratory \\ Parks Road, Oxford OX1 3PU, United Kingdom \\ ${ }^{2}$ Department of Physics, Georgia Southern University \\ Statesboro, GA 30460-8031, USA \\ 3 Physics Laboratory \\ National Institute of Standards and Technology \\ Technology Administration \\ U.S. Department of Commerce \\ Gaithersburg, MD 20899-8410, USA
}

January 21, 2003

\begin{abstract}
We use the Bogoliubov theory of atoms in an optical lattice to study the approach to the Mott-insulator transition. We derive an explicit expression for the superfluid density based on the rigidity of the system under phase variations. This enables us to explore the connection between the quantum depletion of the condensate and the quasi-momentum distribution on the one hand and the superfluid fraction on the other. The approach to the insulator phase may be characterized through the filling of the band by quantum depletion, which should be directly observable via the matter wave interference patterns. We complement these findings by self-consistent Hartree-Fock-Bogoliubov-Popov calculations for onedimensional lattices including the effects of a parabolic trapping potential.
\end{abstract}

\section{Introduction}

Spectacular progress has been made in experimental studies of atoms loaded into an optical lattice in the region of the Mott superfluid insulator quantum phase transition $[1,2]$. In this article, we shall discuss the superfluid density and the quasi-momentum distribution, which is directly related to the matterwave interference patterns that can be observed in such experiments. To do this we use the Bogoliubov method [3], as developed for use in optical lattices [4]. 
In a previous paper [5] we used this method to produce results for squeezing that are consistent with those of other approaches previously reported in the literature $[6,7,8,9]$. In this paper we shall show how it can be used to predict the decrease in the superfluid fraction and the corresponding variations in the matter wave interference fringes that should be directly observable in future experiments. This extends our previous studies based on exact calculation for small one-dimensional systems [10] into the experimentally relevant regime of lattice sizes and particle numbers.

We first introduce the Bose-Hubbard Hamiltonian for atoms in an optical lattice [11]. We then describe briefly our method for determining the superfluid fraction based on the rigidity of the system under a twist of the condensate phase [12]. Using a perturbative formulation analogous to the Drude weight [13] the Bogoliubov approximation gives us a particularly direct way of finding this quantity. It also gives a simple picture of how superfluidity is suppressed by quantum depletion of the condensate. We shall compare the results for various quantities calculated using the Bogoliubov approximation with exact numerical calculations for the case of modest numbers of atoms and lattice sites [10].

\section{The Bose-Hubbard Model and Superfluidity}

The Bose-Hubbard Hamiltonian for atoms in an one-dimensional optical lattice with $I$ sites has the form [11]:

$$
\hat{H}=\sum_{i=1}^{I} \hat{n}_{i} \epsilon_{i}-J \sum_{i=1}^{I}\left(\hat{a}_{i+1}^{\dagger} \hat{a}_{i}+\hat{a}_{i}^{\dagger} \hat{a}_{i+1}\right)+\frac{V}{2} \sum_{i=1}^{I} \hat{n}_{i}\left(\hat{n}_{i}-1\right) .
$$

Here $J$ represents the coupling between adjacent lattice sites due to tunneling and $V$ is the strength of repulsion between atoms on the same site. The noninteracting energy of the atoms on each site, $\epsilon_{i}$, will have some variation that is typically smooth on the scale of the condensate. We shall consider below both, the case where this is a constant, as well as the extension to the case where it varies in a trapped condensate. This Bose-Hubbard Hamiltonian should be an appropriate model when the loading process produces atoms in the lowest vibrational state of each well, with a chemical potential smaller than the distance to the first vibrationally excited state. This is known to be possible from the results of recent experiments $[1,2,14]$.

The concept of superfluidity is closely related to the existence of a condensate in the interacting many-body system. Formally, the one-body density matrix $\rho^{(1)}\left(\vec{x}, \vec{x}^{\prime}\right)$ has to have exactly one macroscopic eigenvalue which defines the number of particles in the condensate; the corresponding eigenvector describes the condensate wave function $\phi_{0}(\vec{x})=e^{i \Theta(\vec{x})}\left|\phi_{0}(\vec{x})\right|$. A spatially varying condensate phase, $\Theta(\vec{x})$, is associated with a velocity field for the condensate by

$$
\vec{v}_{0}(\vec{x})=\frac{\hbar}{m} \vec{\nabla} \Theta(\vec{x}) .
$$


This irrotational velocity field is identified with the velocity of the superfluid flow, $\vec{v}_{s}(\vec{x}) \equiv \vec{v}_{0}(\vec{x})([15],[16])$ and enables us to derive an expression for the superfluid fraction, $f_{s}$. Consider a system with a finite linear dimension, $L$, in the $\vec{e}_{1}$-direction and a ground-state energy, $E_{0}$, calculated with periodic boundary conditions. Now we impose a linear phase variation, $\Theta(\vec{x})=\theta x_{1} / L$ with a total twist angle $\theta$ over the length of the system in the $\vec{e}_{1}$-direction. The resulting ground-state energy, $E_{\theta}$ will depend on the phase twist. For very small twist angles, $\theta \ll \pi$, the energy difference, $E_{\theta}-E_{0}$, can be attributed to the kinetic energy, $T_{s}$, of the superflow generated by the phase gradient. Thus,

$$
E_{\theta}-E_{0}=T_{s}=\frac{1}{2} m N f_{s} \vec{v}_{s}^{2}
$$

where $m$ is the mass of a single particle and $N$ is the total number of particles so that $m N f_{s}$ is the total mass of the superfluid component. Replacing the superfluid velocity, $\vec{v}_{s}$ with the phase gradient according to Eq. (2) leads to a fundamental relation for the superfluid fraction

$$
f_{s}=\frac{2 m}{\hbar^{2}} \frac{L^{2}}{N} \frac{E_{\theta}-E_{0}}{\theta^{2}}=\frac{1}{N} \frac{E_{\theta}-E_{0}}{J(\Delta \theta)^{2}}
$$

where the second equality applies to a lattice system on which a linear phase variation has been imposed. Here the distance between sites is $a$, the phase variation over this distance is $\Delta \theta$, and the number of sites is $I$. In this case, $J \equiv \hbar^{2} /\left(2 m a^{2}\right)$.

Technically the phase variation can be imposed through so-called twisted boundary conditions [12]. In the context of the discrete Bose-Hubbard model it is, however, more convenient to map the phase variation by means of a unitary transformation onto the Hamiltonian. The resulting "twisted" Hamiltonian

$$
\hat{H}_{\theta}=\sum_{i=1}^{I} \hat{n}_{i} \epsilon_{i}-J \sum_{i=1}^{I}\left(e^{-\mathrm{i} \Delta \theta} \hat{a}_{i+1}^{\dagger} \hat{a}_{i}+e^{\mathrm{i} \Delta \theta} \hat{a}_{i}^{\dagger} \hat{a}_{i+1}\right)+\frac{V}{2} \sum_{i=1}^{I} \hat{n}_{i}\left(\hat{n}_{i}-1\right)
$$

exhibits additional phase factors $e^{ \pm \mathrm{i} \Delta \theta}$ - the so-called Peierls phase factors - in the hopping term $[17,18]$. These phase factors show that the twist is equivalent to the imposition of an acceleration on the lattice for a finite time. It is interesting to note that the present experiments enable us to make a specific connection between the formal and operational aspects of the system.

We calculate the change in energy $E_{\theta}-E_{0}$ under the assumption that the phase change $\Delta \theta$ is small so that we can write:

$$
e^{-\mathrm{i} \Delta \theta} \simeq 1-\mathrm{i} \Delta \theta-\frac{1}{2}(\Delta \theta)^{2} .
$$

Using this expansion the twisted Hamiltonian (5) takes the following form:

$$
\hat{H}_{\theta} \simeq \hat{H}_{0}+\Delta \theta \hat{J}-\frac{1}{2}(\Delta \theta)^{2} \hat{T}=\hat{H}_{0}+\hat{H}_{\text {pert }},
$$


where we retain terms up to second order in $\Delta \theta$. The current operator $\hat{J}$ (N.B. that the physical current is given by this expression multiplied by $\frac{1}{\hbar}$ ) and the hopping operator $\hat{T}$ are given by:

$$
\begin{aligned}
& \hat{J}=\mathrm{i} J \sum_{i=1}^{I}\left(\hat{a}_{i+1}^{\dagger} \hat{a}_{i}-\hat{a}_{i}^{\dagger} \hat{a}_{i+1}\right) \\
& \hat{T}=-J \sum_{i=1}^{I}\left(\hat{a}_{i+1}^{\dagger} \hat{a}_{i}+\hat{a}_{i}^{\dagger} \hat{a}_{i+1}\right) .
\end{aligned}
$$

The change in the energy $E_{\theta}-E_{0}$ due to the imposed phase twist can now be evaluated in second order perturbation theory

$$
E_{\theta}-E_{0}=\Delta E^{(1)}+\Delta E^{(2)} .
$$

The first order contribution to the energy change is proportional to the expectation value of the hopping operator

$$
\Delta E^{(1)}=\left\langle\Psi_{0}\left|\hat{H}_{\text {pert }}\right| \Psi_{0}\right\rangle=-\frac{1}{2}(\Delta \theta)^{2}\left\langle\Psi_{0}|\hat{T}| \Psi_{0}\right\rangle .
$$

Here $\left|\Psi_{0}\right\rangle$ is the ground state of the original Bose-Hubbard Hamiltonian (1). The second order term is related to the matrix elements of the current operator involving the excited states $\left|\Psi_{\nu}\right\rangle(\nu=1,2, \ldots)$ of the original Hamiltonian

$$
\Delta E^{(2)}=-\sum_{\nu \neq 0} \frac{\left|\left\langle\Psi_{\nu}\left|\hat{H}_{\text {pert }}\right| \Psi_{0}\right\rangle\right|^{2}}{E_{\nu}-E_{0}}=-(\Delta \theta)^{2} \sum_{\nu \neq 0} \frac{\left|\left\langle\Psi_{\nu}|\hat{J}| \Psi_{0}\right\rangle\right|^{2}}{E_{\nu}-E_{0}} .
$$

Thus we obtain for the energy change up to second order in $\Delta \theta$

$$
\begin{aligned}
E_{\theta}-E_{0} & =(\Delta \theta)^{2}\left(-\frac{1}{2}\left\langle\Psi_{0}|\hat{T}| \Psi_{0}\right\rangle-\sum_{\nu \neq 0} \frac{\left|\left\langle\Psi_{\nu}|\hat{J}| \Psi_{0}\right\rangle\right|^{2}}{E_{\nu}-E_{0}}\right)=I(\Delta \theta)^{2} D \\
D & \equiv \frac{1}{I}\left(-\frac{1}{2}\left\langle\Psi_{0}|\hat{T}| \Psi_{0}\right\rangle-\sum_{\nu \neq 0} \frac{\left|\left\langle\Psi_{\nu}|\hat{J}| \Psi_{0}\right\rangle\right|^{2}}{E_{\nu}-E_{0}}\right) .
\end{aligned}
$$

The quantity $D$, defined above, is formally equivalent to the Drude weight used to specify the DC conductivity of charged fermionic systems [13]. The superfluid fraction is then given by the contribution of both the first and second order term:

$$
\begin{aligned}
f_{\mathrm{s}} & =f_{\mathrm{s}}^{(1)}-f_{\mathrm{s}}^{(2)} \\
f_{\mathrm{s}}^{(1)} & \equiv-\frac{1}{2 N J}\left(\left\langle\Psi_{0}|\hat{T}| \Psi_{0}\right\rangle\right), \\
f_{\mathrm{s}}^{(2)} & \equiv \frac{1}{N J}\left(\sum_{\nu \neq 0} \frac{\left|\left\langle\Psi_{\nu}|\hat{J}| \Psi_{0}\right\rangle\right|^{2}}{E_{\nu}-E_{0}}\right) .
\end{aligned}
$$

Here $N$ is the number of atoms in the lattice. In general both, the first and the second order term contribute. For a translationally invariant lattice the second 
term vanishes (as is going to be shown latter) in the Bogoliubov limit that we shall use in this study. However, in exact calculations and in the Bogoliubov approximation for an inhomogeneous lattice the second order term plays a role.

We can further understand this approach to the superfluid density by calculating the flow that is produced by the application of the phase twist. To do this we work out the expectation value of the current operator expressed in terms of the twisted variables:

$$
\hat{J}_{\theta}=\mathrm{i} J \sum_{i=1}^{I}\left(e^{-\mathrm{i} \Delta \theta} \hat{a}_{i+1}^{\dagger} \hat{a}_{i}-e^{\mathrm{i} \Delta \theta} \hat{a}_{i}^{\dagger} \hat{a}_{i+1}\right) .
$$

We expand this to find the lowest order contributions, i.e.:

$$
\hat{J}_{\theta} \simeq \hat{J}+J \Delta \theta \sum_{i=1}^{I}\left(\hat{a}_{i+1}^{\dagger} \hat{a}_{i}+\hat{a}_{i}^{\dagger} \hat{a}_{i+1}\right)=\hat{J}-\hat{T} \Delta \theta .
$$

We use first order perturbation theory on the wave function to obtain the following expression:

$$
\begin{aligned}
\left\langle\Psi(\Delta \theta)\left|\hat{J}_{\theta}\right| \Psi(\Delta \theta)\right\rangle & =2 \Delta \theta\left(-\frac{1}{2}\left\langle\Psi_{0}|\hat{T}| \Psi_{0}\right\rangle-\sum_{\nu \neq 0} \frac{\left|\left\langle\Psi_{\nu}|\hat{J}| \Psi_{0}\right\rangle\right|^{2}}{E_{\nu}-E_{0}}\right) \\
& =2 N J f_{\mathrm{s}} \Delta \theta .
\end{aligned}
$$

If we note that the kinetic energy for a small quasi-momentum $q$ on a lattice is given by $J q^{2} a^{2}$, we can define the effective mass as $m^{*}=\frac{\hbar^{2}}{2 J a^{2}}$. Here the quasi-momenta are given by $q=\frac{2 \pi}{I a} j$ with $j=1, \ldots,(I-1)$ and lattice spacing a. Thus, the physical current, Eq. (18) multiplied by $\frac{1}{\hbar}$, can be expressed as:

$$
\left\langle\Psi(\Delta \theta)\left|\hat{J}_{\theta}\right| \Psi(\Delta \theta)\right\rangle=N f_{\mathrm{s}} \Delta \theta \frac{\hbar}{m^{*} a^{2}} .
$$

This is the total flux and we need to divide $I$ to get the flux density, i.e.

$$
\begin{aligned}
\frac{1}{I}\left\langle\Psi(\Delta \theta)\left|\hat{J}_{\theta}\right| \Psi(\Delta \theta)\right\rangle & =\left(\frac{\hbar \Delta \theta}{m^{*} a}\right)\left(\frac{N f_{\mathrm{s}}}{a I}\right) \\
& =v_{\mathrm{s}} n_{\mathrm{s}} .
\end{aligned}
$$

So we see that the Drude formulation of the superfluid fraction (14) gives an intuitively satisfying expression for the amount of flowing superfluid.

\section{The Bogoliubov Approximation to the Bose- Hubbard Hamiltonian}

We use the Bogoliubov approximation for the Bose-Hubbard model in the limit that quantum fluctuations, or equivalently depletion of the condensate, is not 
too great. In the limit that the quantum depletion can be completely ignored, we can replace the creation and annihilation operators, $\hat{a}_{i}^{\dagger}$ and $\hat{a}_{i}$, on each site with a $c$-number, $z_{i}$. This leads to a set of coupled non-linear Schrödinger, i.e. Gross-Pitaevskii (GP), equations for these amplitudes [19]:

$$
\mathrm{i} \hbar \partial_{t} z_{i}=-J\left(z_{i+1}+z_{i-1}\right)+V z_{i} z_{i}^{*} z_{i} .
$$

This equation can be used to study the properties of the condensate loaded into the lattice when the tunneling kinetic energy is large enough compared to the interaction energy though small enough for the one-band Bose-Hubbard model to be valid. We then include the quantum fluctuations in our description of the system using the Bogoliubov approximation, where we suppose that we can write the full annihilation operator in terms of the $c$-number part and a fluctuation operator thus:

$$
\hat{a}_{i}=\left(z_{i}+\hat{\delta}_{i}\right) e^{-\mathrm{i} \frac{\mu t}{\hbar}} .
$$

This form will be useful when we are looking at the properties of a timeindependent or adiabatic ground state. In using this method we are assuming that the fluctuation part is small. The Bogoliubov method gives us expressions for the averages of the squares of the fluctuation operator and allows us to determine whether this assumption is valid. We shall examine its validity by comparing the results for various physical quantities with exact numerical calculations based on the Bose-Hubbard Hamiltonian.

\subsection{Bogoliubov theory for the translationally invariant lat- tice}

The ground state solution for the translationally invariant lattice gives the eigenvalue:

$$
\mu=n_{0} V-2 J
$$

where

$$
n_{0}=N / I
$$

is the mean number of atoms on each site of the lattice. We take $N$ to be the

total number of atoms and $I$ to be the number of sites in the one-dimensional lattice.

The Bogoliubov equations for the lattice have the following form:

$$
\mathrm{i} \hbar \partial_{t} \hat{\delta}_{i}=\left(2 n_{0} V-\mu\right) \hat{\delta}_{i}-J\left(\hat{\delta}_{i+1}+\hat{\delta}_{i-1}\right)+n_{0} V \hat{\delta}_{i}^{\dagger} .
$$

This is solved by constructing quasi-particles for the lattice which diagonalize the Hamiltonian [4], i.e

$$
\begin{aligned}
& \hat{\delta}_{i}=\frac{1}{\sqrt{I}} \sum_{q}\left[u^{q} \hat{\alpha}_{q} e^{\mathrm{i}\left(q i a-\omega_{q} t\right)}-v^{q *} \hat{\alpha}_{q}^{\dagger} e^{-\mathrm{i}\left(q i a-\omega_{q} t\right)}\right] \\
& \hat{\delta}_{i}^{\dagger}=\frac{1}{\sqrt{I}} \sum_{q}\left[u^{q *} \hat{\alpha}_{q}^{\dagger} e^{-\mathrm{i}\left(q i a-\omega_{q} t\right)}-v^{q} \hat{\alpha}_{q} e^{\mathrm{i}\left(q i a-\omega_{q} t\right)}\right],
\end{aligned}
$$


where $a$ is the lattice spacing. The quasi-particle operators obey the usual Bose commutation relations:

$$
\left[\hat{\alpha}_{q}, \hat{\alpha}_{q^{\prime}}^{\dagger}\right]=\delta_{q q^{\prime}}
$$

and have the following expectation values at some temperature $T$ :

$$
\left\langle\hat{\alpha}_{q}^{\dagger} \hat{\alpha}_{q^{\prime}}\right\rangle=\delta_{q q^{\prime}}\left[\exp \left(\hbar \omega_{q} / k_{b} T\right)-1\right]^{-1} .
$$

We then find the following equations for the excitation amplitudes and frequencies,

$$
\begin{aligned}
\hbar \omega_{q} u^{q} & =\left[n_{0} V+4 J \sin ^{2}\left(\frac{q a}{2}\right)\right] u^{q}-n_{0} V v^{q}, \\
-\hbar \omega_{q} v^{q} & =\left[n_{0} V+4 J \sin ^{2}\left(\frac{q a}{2}\right)\right] v^{q}-n_{0} V u^{q} .
\end{aligned}
$$

Thus, the expressions for the $u^{q}$ and $v^{q}$ yield:

$$
\begin{aligned}
\left|u^{q}\right|^{2} & =\frac{K(q)+n_{0} V+\hbar \omega_{q}}{2 \hbar \omega_{q}} \\
\left|v^{q}\right|^{2} & =\frac{K(q)+n_{0} V-\hbar \omega_{q}}{2 \hbar \omega_{q}},
\end{aligned}
$$

where the phonon excitation frequencies are given by:

$$
\begin{aligned}
\hbar \omega_{q} & =\sqrt{K(q)\left[2 n_{0} V+K(q)\right]} \\
K(q) & =4 J \sin ^{2}\left(\frac{q a}{2}\right) .
\end{aligned}
$$

\subsection{Expressions for the number superfluid fraction in the translationally invariant lattice}

Having obtained the expressions for the excitations we can now determine the superfluid fraction. The quantity we need to calculate is just the first order term of the Drude expression (14), because the second order term vanishes in the Bogoliubov limit due to the translational invariance of the lattice [see Eq. (48)], i.e.

$$
f_{\mathrm{s}}=-\frac{1}{2 N J}\left\langle\Psi_{0}|\hat{T}| \Psi_{0}\right\rangle=\frac{1}{2 N} \sum_{i=1}^{I}\left\langle\Psi_{0}\left|\hat{a}_{i+1}^{\dagger} \hat{a}_{i}+\hat{a}_{i}^{\dagger} \hat{a}_{i+1}\right| \Psi_{0}\right\rangle .
$$

In the Bogoliubov approximation this has the form:

$$
\begin{aligned}
f_{\mathrm{s}} & =\frac{1}{2 N} \sum_{i=1}^{I}\left\langle\Psi_{0}\left|\left(\hat{\delta}_{i+1}^{\dagger}+z_{i+1}\right)\left(\hat{\delta}_{i}+z_{i}\right)+\left(\hat{\delta}_{i}^{\dagger}+z_{i}\right)\left(\hat{\delta}_{i+1}+z_{i+1}\right)\right| \Psi_{0}\right\rangle \\
& =\frac{1}{2 N} \sum_{i=1}^{I}\left\langle\Psi_{0}\left|2 z_{i}^{2}+\hat{\delta}_{i+1}^{\dagger} \hat{\delta}_{i}+\hat{\delta}_{i}^{\dagger} \hat{\delta}_{i+1}\right| \Psi_{0}\right\rangle .
\end{aligned}
$$


We can now express the fluctuation operators, Eqs. (26) and (27), in terms of the quasi-particle operators that diagonalize the quadratic Hamiltonian. This leads to the following expression for the superfluid fraction at finite temperature:

$$
\begin{aligned}
f_{\mathrm{s}}=\frac{1}{2 N}[ & \sum_{i=1}^{I} 2 z_{i}^{2}+\frac{1}{I}\left\langle\sum_{q}\left[u^{q} \hat{\alpha}_{q} e^{\mathrm{i} q(i+1) a}-v^{q} \hat{\alpha}_{q}^{\dagger} e^{-\mathrm{i} q(i+1) a}\right]\right. \\
& \left.\times \sum_{q^{\prime}}\left[u^{q^{\prime}} \hat{\alpha}_{q^{\prime}}^{\dagger} e^{-\mathrm{i} q^{\prime} i a}-v^{q^{\prime}} \hat{\alpha}_{q^{\prime}} e^{\mathrm{i} q^{\prime} i a}\right]\right\rangle \\
& +\frac{1}{I} \sum_{i=1}^{I}\left\langle\sum_{q}\left[u^{q} \hat{\alpha}_{q}^{\dagger} e^{-\mathrm{i} q i a}-v^{q} \hat{\alpha}_{q} e^{\mathrm{i} q i a}\right]\right. \\
& \left.\left.\times \sum_{q^{\prime}}\left[u^{q^{\prime}} \hat{\alpha}_{q^{\prime}} e^{\mathrm{i} q^{\prime}(i+1) a}-v^{q^{\prime}} \hat{\alpha}_{q^{\prime}}^{\dagger} e^{-\mathrm{i} q^{\prime}(i+1) a}\right]\right\rangle\right]
\end{aligned}
$$

and we find in the zero temperature limit of a translationally invariant lattice:

$$
f_{\mathrm{s}}=\frac{I}{N}\left[z^{2}+\frac{1}{I} \sum_{q}\left|v^{q}\right|^{2} \cos (q a)\right] .
$$

Here the summation runs over all quasi-momenta $q=\frac{2 \pi}{I a} j$ with $j=1, \ldots,(I-1)$ and we have called $z$ the value of all $z_{i}$ in a translationally invariant system. This shows that in the limit of zero lattice spacing (while keeping $q$ finite) the superfluid fraction is unity as we have the normalization condition:

$$
I z^{2}+\sum_{q}\left|v^{q}\right|^{2}=N .
$$

These expressions give a direct insight into the change of the superfluid fraction as atoms are pushed out of the condensate due to interactions. In Eq. (39) the sum involving the Bogoliubov amplitudes $v^{q}$ characterizes the difference between the condensate fraction, which is given by the first term, and the superfluid fraction. For weak interactions and a small depletion, which fills only the lower quarter of the band where the $\cos (q a)$ term has a positive sign, the superfluid fraction is larger than the condensate fraction. Thus the depletion of the condensate has initially little effect on superfluidity. When the depleted population spreads into the central part of the band, where the $\cos (q a)$ term has a negative sign, the superfluid fraction is reduced and might even become smaller than the condensate fraction. Finally, the population in the upper quarter of the band again produces a positive contribution to the superflow. In a sense the interactions are playing a role akin to Fermi exclusion "pressure" in the case of electron flow in a band. This, however can lead to perfect filling and cancellation of the flow. In the case of our Bogoliubov description we can only see reduction of the flow, not a perfect switching off of the superfluid. This happens in the Mott insulator state, which cannot be described by the Bogoliubov approximation. 
In the next section we outline the version of the Bogoliubov theory that should be best suited to treating these problems, i.e self-consistent Bogoliubov theory.

\section{Self-consistent HFB-Popov theory}

In this section we explore the limits of validity of the simplest zero temperature self-consistent Bogoliubov theory, a simplified version of the Hartree-FockBogoliubov approximation originally introduced by Popov [20]. The HFB-Popov theory is an extension of the standard Bogoliubov approximation that takes into account the depletion of the condensate but neglects the anomalous average. As discussed in the previous section, taking into account the depletion of the condensate is important as the transition is approached because the depleted population causes the reduction of the superfluidity. Although the HFB-Popov aproach has the limitation that it doesn't take into account the full effect of the medium because it neglects the anomalous average [21], it can be considered a better theory for the elementary excitations than the full HFB due to the fact that the theory is gapless and doesn't violate Goldstone's theorem.

A derivation of the Bogoliubov equations for the quasiparticle amplitudes in a lattice can be found for example in Ref. [5]. These equations only take into account terms up to second order in the fluctuations. Including third and fourth order terms by treating them in a self-consistent mean field approximation ( [22], [23] ) and neglecting anomalous average terms yields the following HFB-Popov equations:

$$
\begin{aligned}
\hbar \omega_{q} u_{i}^{q}+c^{q} z_{i} & \left.=\left(2 V\left(\left|z_{i}\right|^{2}+\tilde{n}_{i}\right)-\mu+\epsilon_{i}\right) u_{i}^{q}-J\left(u_{i+1}^{q}+u_{i-1}^{q}\right)-V z_{i}^{2} v_{\alpha}^{q} 41\right) \\
-\hbar \omega_{q} v_{i}^{q}-c^{q} z_{i}^{*} & \left.=\left(2 V\left(\left|z_{i}\right|^{2}+\tilde{n}_{i}\right)-\mu+\epsilon_{i}\right) v_{i}^{q}-J\left(v_{i+1}^{q}+v_{i-1}^{q}\right)-V z_{i}^{* 2} u_{i}^{q}, 2\right) \\
\mu z_{i} & =-J\left(z_{i+1}+z_{i-1}\right)+\left(V\left(\left|z_{i}\right|^{2}+2 \tilde{n}_{i}\right)+\epsilon_{i}\right) z_{i} \\
\tilde{n}_{i} & =\sum_{q}\left|v_{i}^{q}\right|^{2} \\
N & =\sum_{i=1}^{I}\left(\left|z_{i}\right|^{2}+\tilde{n}_{i}\right) \\
c^{q} & =V \sum_{i}\left|z_{i}\right|^{2}\left(z_{i}^{*} u_{i}^{q}-z_{i} v_{i}^{q}\right)
\end{aligned}
$$

Where $\epsilon_{i}$ is the energy offset at site $i$ due to an external potential, $\left\{u_{i}^{q}, v_{i}^{q}\right\}$ and $\omega_{q}$ are respectively the quasiparticle amplitudes and energies, thus

$$
\hat{\delta}_{i}=\sum_{q} u_{i}^{q} \hat{\alpha}_{q} e^{-\mathrm{i} \omega_{q} t}-v_{i}^{q *} \hat{\alpha}_{q}^{\dagger} e^{\mathrm{i} \omega_{q} t}
$$

$\tilde{n}_{i}$ is the depletion at site $i$, and $N$ is the total number of particles. The parameters $c^{q}$ ensure the $\left\{u_{i}^{q}, v_{i}^{q}\right\}$ solutions to the above equations with $\omega_{q} \neq 0$ to be orthogonal to the condensate (Ref. [23]). 
By calculating the quasiparticle amplitudes and the condensate density it is possible to get information about most of the physical properties of the system. For example the superfluid fraction and the on site number fluctuations are given by:

$$
\begin{aligned}
& f_{\mathrm{s}}=f_{s}^{(1)}-f_{s}^{(2)}, \\
& f_{s}^{(1)}=\sum_{i=1}^{I} f_{s i}^{(1)}=\frac{1}{2 N} \sum_{i=1}^{I}\left[\left(z_{i+1} z_{i}^{*}+z_{i+1}^{*} z_{i}\right)+\sum_{q}\left(v_{i}^{q} v_{i+1}^{q *}+v_{i}^{q *} v_{i+1}^{q}\right)\right], \\
& f_{s}^{(2)}=\frac{J}{N}\left(\sum_{q, q^{\prime}} \frac{\left|\sum_{i}\left(u_{i+1}^{q} v_{i}^{q^{\prime}}-u_{i}^{q} v_{i+1}^{q^{\prime}}\right)\right|^{2}}{\hbar \omega_{q}+\hbar \omega_{q^{\prime}}}+\delta_{q q^{\prime}} \frac{\left|\sum_{i}\left(u_{i+1}^{q} v_{i}^{q}-u_{i}^{q} v_{i+1}^{q}\right)\right|^{2}}{2 \hbar \omega_{q}}\right), \\
& \Delta n_{i}^{2}=\left|z_{i}\right|^{2} \sum_{q}\left|u_{i}^{q}-v_{i}^{q *}\right|^{2} .
\end{aligned}
$$

From the complete expression of the superfluid fraction, it can be seen explicitly how due to the translational invariance, the second order term vanishes in the homogeneous system.

\subsection{Translationally Invariant lattice}

For the translational invariant lattice we use the quasiparticle transformation given by Eqs. (26) and (27). Under this transformation the self consistent equations can be written, generalizing the previous version, as:

$$
\begin{aligned}
\mu & =\left(|z|^{2}+\frac{2}{I} \sum_{q}\left|v^{q}\right|^{2}\right) V-2 J \\
\left|u^{q}\right|^{2} & =\frac{K(q)+|z|^{2} V+\hbar \omega_{q}}{2 \hbar \omega_{q}} \\
\left|v^{q}\right|^{2} & =\frac{K(q)+|z|^{2} V-\hbar \omega_{q}}{2 \hbar \omega_{q}} \\
N & =I|z|^{2}+\sum_{q}\left|v^{q}\right|^{2}
\end{aligned}
$$

Here the phonon excitation spectrum is given by:

$$
\hbar \omega_{q}=\sqrt{K(q)\left[2|z|^{2} V+K(q)\right]}
$$

and $K(q)$ is given by Eq. (35). Again we omit the subscript in the amplitudes $z_{i}$ because they have the same value at all lattice sites. Notice also that due to translational invariance, the $c^{q}$ coefficients vanish.

In the homogeneous system, the form of the HFB-Popov equations for the quasiparticle amplitudes and energies is very close to the standard Bogoliubov form. We do, however, have to replace $n_{0}=N / I$ by the condensate amplitude $|z|^{2}$ which must take into account the depletion of the condensate. We solve 
for the condensate amplitude as a function of the external parameters $J, V, N$ and $I$ by inserting Eq. (52) in Eq. (53). Once $|z|^{2}$ is determined, we use it to calculate the other expressions.

In Fig. 1 we compare the number fluctuations on a lattice site, the condensate fraction and the total and second order superfluid fraction determined from the exact solution of the Bose-Hubbard Hamiltonian to the self consistent HFB-Popov predictions as a function of the ratio $V_{\text {eff }}=V / J$. The systems used for the comparisons have three wells, $I=3$, and commensurate filling factors $n_{0}=5,10,20$ and 50. Similar results for the incommensurate case with $N=16,31,61,151$ are shown in Fig. 2. We were restricted to consider only three wells due to computational limitations. The size of the matrix needed in the exact solution for $N$ atoms and $I$ wells scales as $\frac{(N+I-1) !}{N !(I-1) !}$. However, if the HFB-Popov approach works well for these small systems we expect it to provide a good description of the larger systems prepared in the lab.

Because the second order term of the superfluid fraction (second term of Eq.14) vanishes in the HFB-Popov approach (see Eq. 48), we only expect the self consistent HFB-Popov theory to give a good description of the superfluid fraction in the region where the second order term is extremely small, provided it predicts accurately the first order term. This is exactly what is observed in the

plots. When the second order term starts to grow, typically above $0.5 V_{e f f}^{\text {crit }}$, the HFB-Popov theory starts to fail. An estimate of $V_{e f f}^{\text {crit }}$ is shown by a vertical line in some of the figures. This was obtained by using the second order perturbative approach presented in Ref. [4]. With increasing filling factor the critical value is shifted towards larger values of the interaction strength, and the region in which the HFB-Popov theory is accurate gets larger. It is interesting to note that the number fluctuations predicted by the theory are accurate in a greater range than the other physical quantities shown. Its predictions of squeezing agree very well with the exact solutions right up to the point where the number fluctuations become less than unity.

For the cases with non-commensurate fillings depicted in Fig. 2, the agreement is significantly better for all quantities. This is not surprising because when the filling is not commensurate there is always a superfluid present and the Mott transition doesn't occur. As can be seen in the plots for these cases the second order term is always very small.

\subsection{Inhomogeneous lattice}

In this section we consider the experimentally relevant case when there is an external magnetic confinement in addition to the lattice potential. In this situ- 

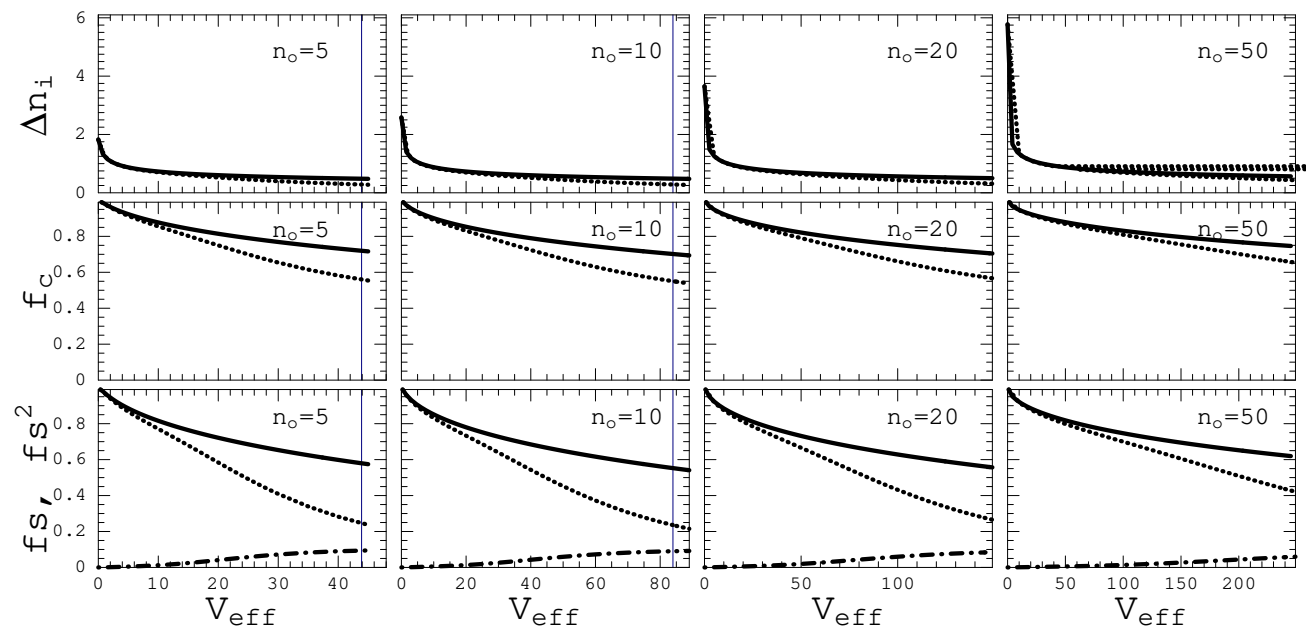

Figure 1: Comparisons of the exact solution (dotted line) and HFB-Popov (solid line) as a function of $V_{\text {eff }}=V / J$, for a system with $I=3$ and filling factors $n_{o}=5,10,20$, and 50. Top: number fluctuations, middle: condensate fraction, bottom: superfluid fraction $f_{\mathrm{s}}$. The exact second order term (dashed line) of the superfluid fraction, $f_{\mathrm{s}}^{(2)}$ is also shown in these plots. The vertical line shown in some plots is an estimation of $V_{e f f}^{c r i t}$.

ation, the self consistent HFB-Popov equations take the form:

$$
\begin{aligned}
\hbar \omega_{q} u_{i}^{q}+c^{q} z_{i} & =\left(2 V\left(\left|z_{i}\right|^{2}+\tilde{n}_{i}\right)-\mu+\Omega i^{2}\right) u_{i}^{q}-J\left(u_{i+1}^{q}+u_{i-1}^{q}\right)-V z_{i}^{2} v_{i}^{q}(55) \\
-\hbar \omega_{q} v_{i}^{q}-c^{q} z_{i}^{*} & \left.=\left(2 V\left(\left|z_{i}\right|^{2}+\tilde{n}_{i}\right)-\mu+\Omega i^{2}\right) v_{i}^{q}-J\left(v_{i+1}^{q}+v_{i-1}^{q}\right)-V z_{i}^{* 2} u u_{i}^{q} 56\right) \\
\mu z_{i} & =-J\left(z_{i+1}+z_{i-1}\right)+\left(V\left(\left|z_{i}\right|^{2}+2 \tilde{n}_{i}\right)+\Omega i^{2}\right) z_{i} \\
\tilde{n}_{i} & =\sum_{q}\left|v_{i}^{q}\right|^{2} \\
N & =\sum_{i}\left(\left|z_{i}\right|^{2}+\tilde{n}_{i}\right) \\
c^{q} & =V \sum_{i}\left|z_{i}\right|^{2}\left(z_{i}^{*} u_{i}^{q}-z_{i} v_{i}^{q}\right)
\end{aligned}
$$

where $\Omega=\frac{1}{2} m \omega^{2} a^{2}$, with $m$ the mass of the atoms, $\omega$ the trap frequency, and $a$ the lattice spacing. The site indices $i$ are chosen such that $i=0$ corresponds to the center of the trap. Again the $c^{q}$ are introduced to ensure the orthogonality of the excitations to the condensate [23]. We have solved the HFB-Popov equations 

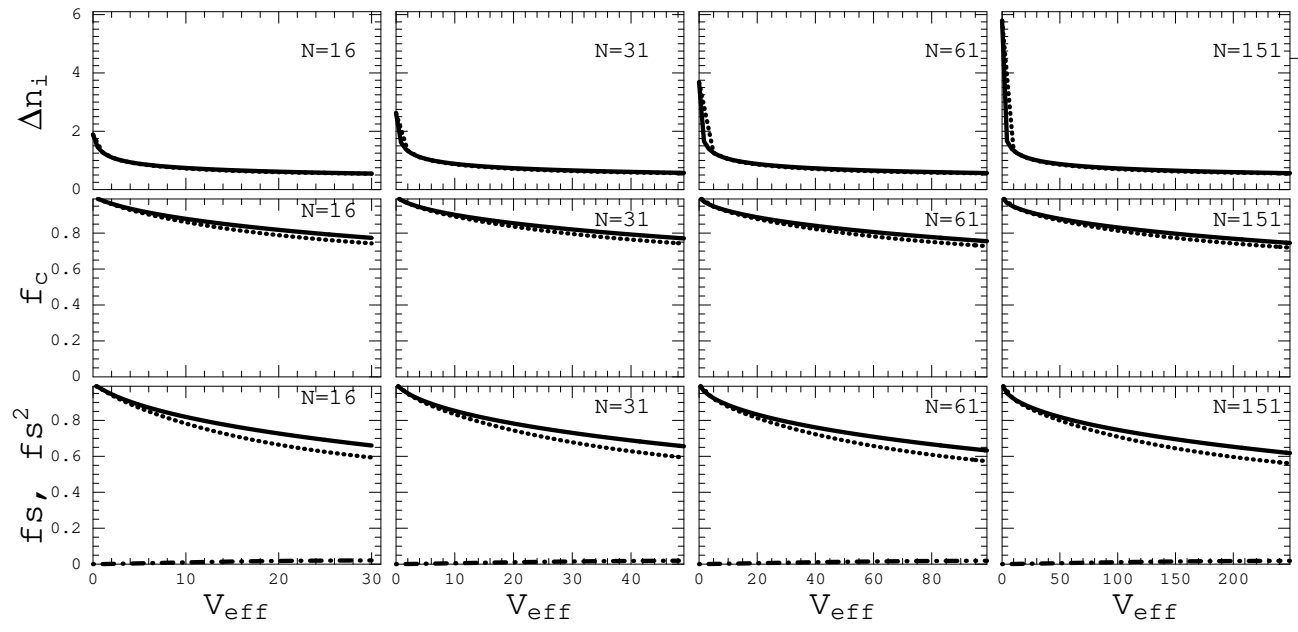

Figure 2: Comparisons of the exact solution (dotted line) and HFB-Popov (solid line) for a system with $I=3$ and non commensurate filling factors $N=16,31,61,151$ as a function of $V_{e f f}=V / J$. Top: number fluctuations, middle: condensate fraction, bottom: superfluid fraction $f_{\mathrm{s}}$. In these plots the exact second order term of the superfluid fraction is also shown with a dashed line.

for this system by an iterative procedure, similar to the one followed in Ref. [24]. Each cycle of the iteration consists of two steps. In the first step we solve Eq. (57) subject to the constraint Eq. (59) by using the $\tilde{n}_{i}$ obtained in the previous cycle. This generates new values for the $z_{i}$. In the second step we solve for $\left\{u_{i}^{q}, v_{i}^{q}\right\}$ in Eqs. (55) using the $\tilde{n}_{i}$ from the previous cycle and the newly generated $z_{i}$. The $\left\{u_{i}^{q}, v_{i}^{q}\right\}$ are used then to update $\tilde{n}_{i}$. Because the HFBPopov is gapless, it is possible to keep the orthogonality of the excitations to the condensate by solving Eqs. (55) with the $c^{q}$ set to zero but removing in each cycle the projection of the calculated $\left\{u_{i}^{q}, v_{i}^{q}\right\}$ amplitudes onto the condensate. Convergence is reached when the change in $\sum_{i}\left|\tilde{n}_{i}\right|^{2}$ from one cycle to the next is smaller than a specified tolerance.

The parameters chosen for the numerical calculations were $\Omega=0.0015 E_{R}$, with $E_{R}$ the one photon recoil energy, which for the case of a Rubidium condensate corresponds to a trap frequency of approximately $90 \mathrm{~Hz}$. We used a total number of 1000 atoms, $N=1000$, and set $V N=1.0 E_{R} . J$ was varied to achieve a range of $V_{\text {eff }}=V / J$ between 0.01 and 312. The range was chosen based on a local mean field approach [4], which for our parameters estimates 
the transition region between $V_{\text {eff }} \approx 640$ (at the center where the local filling factor is approximately 80 ) and $V_{\text {eff }} \approx 12$ (at the wings).

The results of the numerical calculations are summarized in Figs. 3 to 8 . In Fig. 3 we plot the evolution of the density profile (black boxes), the condensate population (triangles) and the on-site depletion (empty diamonds) as $V_{\text {eff }}$ is increased. In the plots we also show, for comparison purposes, the ground state density profile for $J=0$ (empty boxes). This has the advantage that can be calculated exactly from the Hamiltonian. In general we observe the reduction of the condensate population and thus the increment of the depletion as the interaction strength is increased. When the system is in the superfluid regime most of the atoms are in the condensate but as $J$ is decreased the depletion of the condensate becomes very important.

For the chosen parameters, the density profile has a parabolic shape reflecting the confining potential. By comparing the evolution of the density as $J$ is decreased with the exact solution at $J=0$, we can crudely estimate the validity of the HFB-Popov calculations. The density evolves from a Gaussian type (see plots for $V_{e f f}=0.01$ and 0.09 ) with smooth edges towards a Thomas-Fermi profile with sharp edges adjusting its shape to the $J=0$ profile. We can appreciate that around $V_{\text {eff }}=3$ both profiles are almost equal. For lower values of $J$ the HFB-Popov density starts to differ from the $J=0$ one, even though the system is closer to the $J=0$ limit. We can say that beyond this point higher order correlations, neglected by the theory, begin to be important. The departure of the HFB-Popov density profile from the $J=0$ one as $J$ is decreased begins at the edges (see panel corresponding to $V_{\text {eff }}=11$ and 100). This is something expected if we look at the on-site depletion. For such values of $V_{\text {eff }}$ the local depletion at the wings corresponds to a considerable percentage of the condensate populations, and thus the validity of the HFBPopov assumptions starts to be dubious. The homogeneous results shown in the previous section corroborate our present statements for the confined system. For the smallest filling factor (see Fig.1) the differences between the homogeneous HFB-Popov calculations and the exact solutions become important for values of $V_{\text {eff }}$ greater than 20. For higher values of $V_{\text {eff }}$, see plot for $V_{\text {eff }}=312$, the HFB-Popov density predictions differs from the $J=0$ solution even at the central wells. At this point the failure of the method is clear and a fully quantal method is required.

The HFB-Popov quasiparticle spectrum is shown in Fig.4. It can be observed how the lower energy eigenvalues evolve from a linear non degenerated spectrum to an almost degenerated one as $\mathrm{J}$ is decreased. It is worth it to mention that the small energy difference between the ground and first excited states for high values of $V_{\text {eff }}$ makes the numerical solution very unstable in the sense that it was very easy to jump to an excited state when solving for the condensate wave function. The decrement in the energy spacing predicted by the HFB-Popov theory as the system approaches the transition is very useful to keep in mind 
for the experimental realization of the Mott transition. As the optical lattice depth is ramped up the adiabaticity criteria is harder to fulfill.

In Fig. 5 we plot the results for the number fluctuations found numerically using the inhomogeneous HFB-Popov approach. The number fluctuations profile reflects the condensate profile. We also show the number fluctuations evaluated by using a local density approximation (empty boxes). The latter was calculated by substituting in the number fluctuations expression (Eq. 49) the $\left\{u^{q}, v^{q}\right\}$ amplitudes found for the homogeneous system (Eqs. 51 and 52), but replacing the condensate density in each lattice site by the one found numerically for the trapped system (see Fig. 3). The complete agreement between the two approaches justifies the validity of the local density approximation for the estimations of local quantities in confined systems. Based on this agreement and the results for the homogeneous system shown in the previous section, we expect that the inhomogeneous HFB-Popov results for squeezing also agrees with the exact solution right up to the transition.

\subsection{Quasi-momentum distribution in the inhomogeneous lattice}

The quasi-momentum distribution of the atoms released from the lattice is important because it is one of the most easily accessible quantities to the experiments. The quasi-momentum distribution function $n_{q}$ is defined as [10]

$$
n_{q}=\sum_{i, j} e^{\mathrm{i} q(i-j) a}\left\langle a_{i}^{\dagger} a_{j}\right\rangle,
$$

where the quasi-momentum $q$ can assume discrete values which are integer multiples of $\frac{2 \pi}{I a}, a$ is the lattice spacing. In Fig. 6 we present the quasi-momentum distribution for the same parameters used in the previous section. The distribution for the two lowest values of $V_{\text {eff }}$ corresponds to the one that characterizes an uncorrelated superfluid phase with a narrow peak at small quasi-momenta. As the hopping rate is decreased we observe that the sharpness of the central peak decreases and the distribution extends towards large quasi-momenta. It is interesting to note the appearance of a small peak between $q=0.5$ and 1 which is most noticeable for the $V_{\text {eff }}=3$ case. This agrees with the results found in [25] where they solve numerically the Bose-Hubbard Hamiltonian by using Monte Carlo simulations. We attribute the origin of the small peak to the depletion of the condensate at the wings. For the parameters when the small peak is present, the most important contribution to the quasi-momentum distribution still comes from the condensate atoms. The step function like shape of the condensate profile causes an oscillatory $|\sin (x) / x|$ shape of the quasi-momentum distribution. As the lattice depth is increased the hopping becomes energetically costly, the long-range order starts to decrease and the Fourier spectrum becomes broader. 
In Fig. 7 we plot the first order on site superfluid fraction $f_{s i}^{(1)}$ which was defined in Eq.(48). The curves corresponding to $V_{\text {eff }}=0.01-11$, which are in the regime where the HFB-Popov is expected to be valid, depict how as $V_{\text {eff }}$ is increased the superfluid profile decreases faster at the wings and at the center but no major change is observed in the middle section. The evolution of the on-site superfluidity as the interaction strength is increased, exhibiting a domain localized decrement instead of a global one, is in agreement with the development of uncompressible regions surrounded by superfluid rings predicted for trapped systems [26] as the transition is approached.

Fig. 8 shows the first order and total superfluid fraction and also the second order superfluid fraction as a function of $V_{\text {eff }}$. Different from the translationally invariant case, the second order contribution calculated in the HFB-Popov approach doesn't vanish for the inhomogeneous system. The rapid decrement of the superfluid fraction observed after $\log \left(V_{e f f}\right) \sim 1.2$ is a signature that the system is entering a highly correlated regime. Beyond this point higher order correlations neglected in the HFB-Popov approach become crucial and a more accurate approach is required.

The Mott transition is a quantum phase transition and as all critical phenomena its behavior depends strongly on the dimensionality of the system. In the present analysis, due to computational limitations, we considered one dimensional systems. Experimentally, the Mott transition has been achieved [2] in a 3 dimensional lattice with filling factors between 1 and 3. Even though the HFB-Popov approach fails to describe the strong coupling regime for the one dimensional systems we considered in the present paper, we showed how the method is incredibly powerful in describing most of its characteristic features as they are driven from the superfluid regime towards the transition. We expect the HFB-Popov method to give a better description of the transition as the dimensionality of the system is increased and therefore to be a good model in an experimental situation.

As shown in previous studies [27], [28] the Mott transition in a d-dimensional homogeneous system has two different critical behaviors: one $(\mathrm{d}+1) \mathrm{XY}$ - like, for systems with fixed integer density as the interaction strength is changed, and one mean field-like exhibited when the transition is induced by changing the density. Different from the homogeneous case where the Mott transition is characterized by the global offset of the superfluidity, for confined systems, commensuration is only well defined locally. The inhomogeneity introduced by the confined potential allows the existence of extended Mott domains (above a critical interaction strength) surrounded by superfluid ones [26], thus the total superfluid fraction doesn't vanishes in the Mott regime. This issue, together with the fact that the finite length scale introduced by the trap suppresses the long wave fluctuations which are responsible for destroying the mean field $[23]^{1}$, make us believe the critical behavior in confined systems to be more mean-field like. Because the critical dimension for the latter type of transition is two [27],

\footnotetext{
${ }^{1}$ One obvious consequence of this is that $\mathrm{BEC}$ is possible in one and two dimensions in a trap whereas in the homogeneous, thermodynamic limit it can not occur in fewer than three dimensions
} 
[28], we expect that for trapped systems in $d=3$, the range of validity of the HFB-Popov extends closer to the transition.

\section{Summary}

We have developed in this article a Bogoliubov method for describing the approach of a condensate loaded in an optical lattice towards the Mott transition. We have shown that this method can be used to predict the relevant physical quantities over a useful range. We have also shown how it gives a powerful insight into the way quantum depletion reduces the long range order and the superfluid fraction.

\section{Acknowledgments}

This work was supported in part by US National Science Foundation grants PHY-0100634 and PHY-0100767, the United Kingdom's Engineering and Physical Sciences Research Council, the Cold Quantum Gases Network Research

Training Network, and the Advanced Research and Development activity.

\section{References}

[1] C. Orzel, A. K. Tuchman, M. L. Fenselau, M. Yasuda and M. A. Kasevich "Squeezed states in a Bose-Einstein Condensate" Science 291, 2386 (2001)

[2] M. Greiner, O. Mandel, T. Esslinger, T. W. Hänsch, and I. Bloch, "Quantum phase transition from a superfluid to a Mott insulator in a gas of ultracold atoms," Nature 415, 39 (2002)

[3] N. N. Bogoliubov, "On the theory of superfluidity" J. Phys. (USSR) 11, $23(1947)$

[4] D. van Oosten, P. van der Straten, H. T. C. Stoof, "Quantum phases in an optical lattice" Phys. Rev. A 63, 053601 (2001)

[5] K. Burnett, M. Edwards, C. W. Clark and M. Shotter, "The Bogoliubov approach to number squeezing in an optical lattice", J. Phys. B: At. Mol. Opt. Phys. 35, 1671 (2002).

[6] J. Javanainen and M. Yu. Ivanov, "Splitting a trap containing a BoseEinstein condensate: atom number fluctuations," Phys. Rev. A 60, 2351(1999)

[7] J. Javanainen, "Phonon approach to an array of traps containing BoseEinstein condensates," Phys. Rev. A 60, 4902 (1999)

[8] M. Shotter, " A Gutzwiller approach to squeezed states in an optical lattice," J. Phys. B: At. Mol. Opt. Phys. 35, 3019-3028 (2002) 
[9] G. Baym, "Topics in the microscopic theory of Bose-Einstein condensates," J. Phys. B: At. Mol. Opt. Phys. 34, 4541-4550 (2001)

[10] R. Roth, K. Burnett, "Superfluidity and interference pattern of ultracold bosons in optical lattices", arXiv:cond-mat/0209066 (2002)

[11] D. Jaksch, C. Bruder, C. W. Gardiner, J.I. Cirac and P. Zoller, "Cold bosonic atoms in optical lattices," Phys. Rev. Lett. 81, 3108 (1998)

[12] M. E. Fisher, M. N. Barber, and D. Jasnow, "Helicity modulus, superfluidity, and scaling in isotropic systems," Phys. Rev. A 8, 1111 (1973)

[13] B. S. Shastry and B. Sutherland, "Insulator, metal or superconductor: The criteria," Phys. Rev. B 47, 7995 (1993)

[14] S. Rolston et al (private communication)

[15] A. J. Legett, "Superfluidity," Rev. Mod. Phys. 71, S318 (1999)

[16] E. M. Lifshitz and L. Pitaevskii, Statistical Physics part 2, Pergamon (Oxford, 1980)

[17] D. Poilblanc, "Twisted boundary conditions in cluster calculations of the optical conductivity in two-dimensional lattice models," Phys. Rev. B 44, $9562(1991)$

[18] B. S. Shastry and B. Sutherland, "Twisted boundary conditions and effective mass in Heisenberg-Ising and Hubbard rings," Phys. Rev. Lett. 65, $243(1990)$

[19] L. Amico and V. Penna, "Time dependent mean-field theory of superfluidinsulator phase transition," Phys. Rev. B 62, 1224 (2000)

[20] V. N. Popov, Functional Integrals and Collective Modes, (Cambridge University Press, New York 1987)

[21] N.P. Proukakis, S.A. Morgan, S. Choi and K. Burnett, "Comparison of gapless mean-field theories for trapped Bose-Einstein condensates, " Phys. Rev. A 58, 2435 (1998)

[22] A. Griffin, "Conserving and gapless approximation for an inhomogeneous approximation Bose gas at finite temperature," Phys. Rev. B, 53, 9341 (1995).

[23] S. A. Morgan, A gapless theory of Bose-Einstein Condensation in Dilute Gases at Finite Temperature, D. Phil. Thesis, Oxford University (unpublished, 1999)

[24] R. J. Dodd, M. Edwards, C. W. Clark and K. Burnett, "Collective excitations of Bose-Einstein-condensed gases at finite temperature," Phys. Rev. A, 57, R32 (1998). 
[25] V. A. Kashurnikov, N. V. Prokof'ev and B. V. Svistunov, "Revealing superfluid-Mott-insulator transition in an optical lattice," arXiv:condmat/0202510 (2002).

[26] G. G. Batrouni et al.," Mott domains of bosons cofined on optical lattices", Phys. Rev. Lett, 89, 117203 (2002).

[27] M. P. A. Fisher, P. B. Weichman, G. Grinstein and D. S. Fisher, "Boson localization and the superfluid-insulator transition," Phys. Rev. B,40, 546 (1989).

[28] G. G. Batrouni, R. T. Scalettar and G. T. Zymanyi, "Quantum critical phenomena in one-dimensional Bose systems," Phys. Rev. Lett. 65, 1765 (1990). 


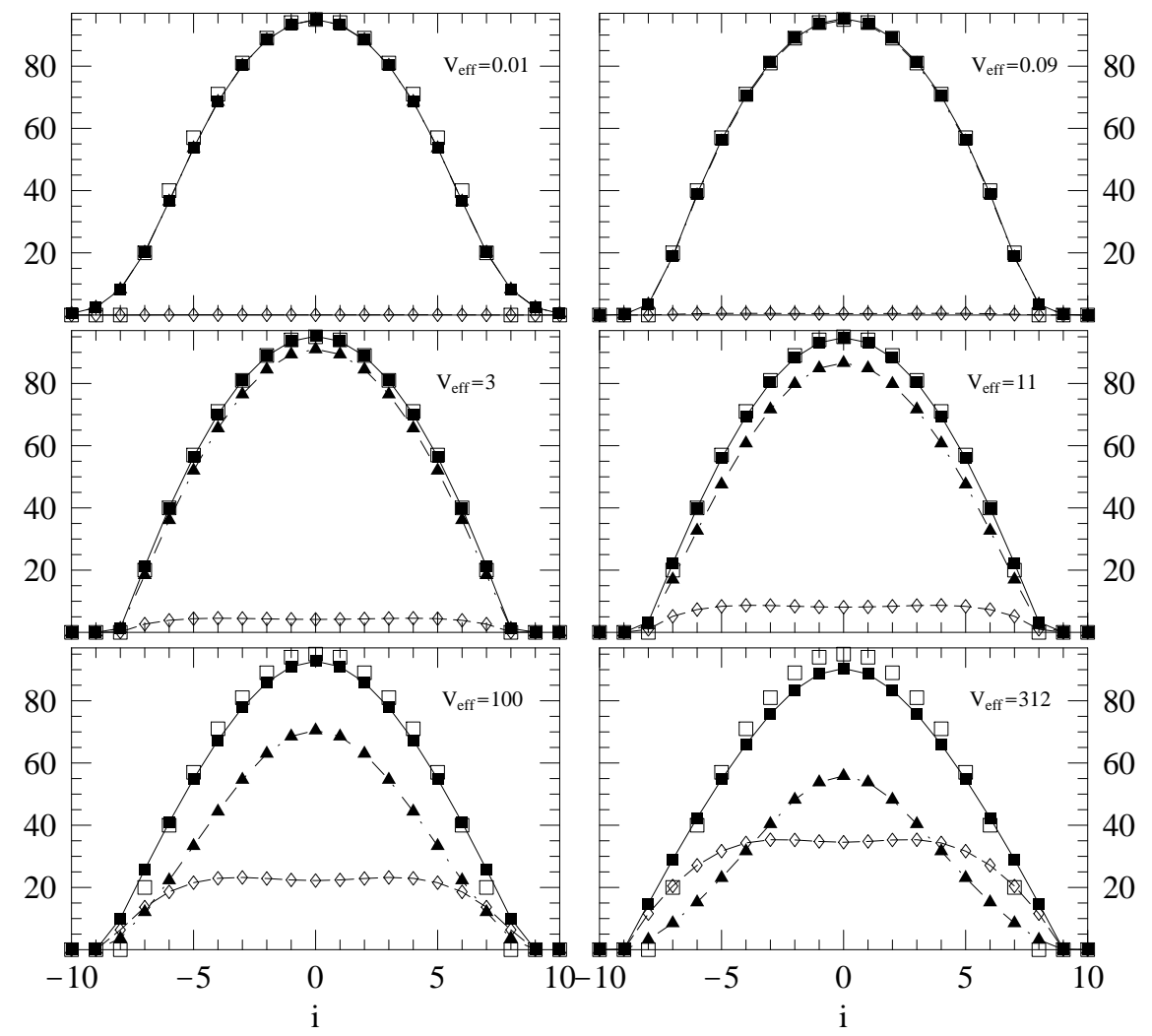

Figure 3: Condensate density (triangles), total density (filled boxes) and local depletion (empty diamonds) as a function of the lattice site for different values of $V_{\text {eff. }}$. Although these quantities are defined only at the discrete lattice sites we join them to help visualization. The empty boxes represent the exact solution for the case $\mathrm{J}=0$. 


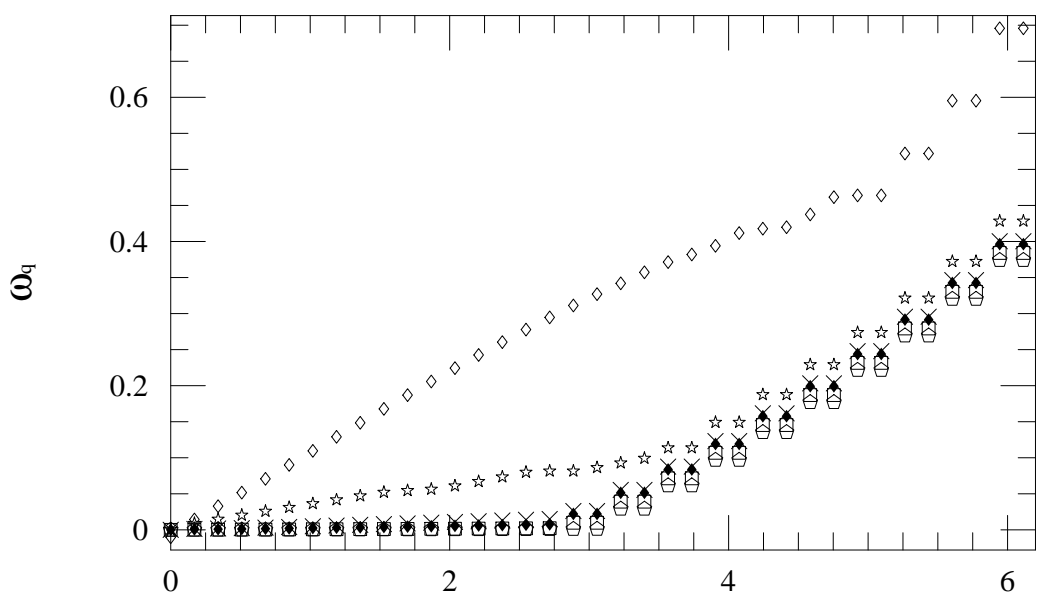

q

Figure 4: Quasiparticle spectrum in ascending order predicted by the HFBPopov theory for different values of $V_{\text {eff }}$ : Empty diamonds $\left(V_{\text {eff }}=0.01\right)$, stars $\left(V_{\text {eff }}=0.09\right)$, crosses $\left(V_{\text {eff }}=3\right)$, filled diamonds $\left(V_{\text {eff }}=11\right)$, empty boxes $\left(V_{e f f}=100\right)$ and polygons $\left(V_{\text {eff }}=312\right)$ 


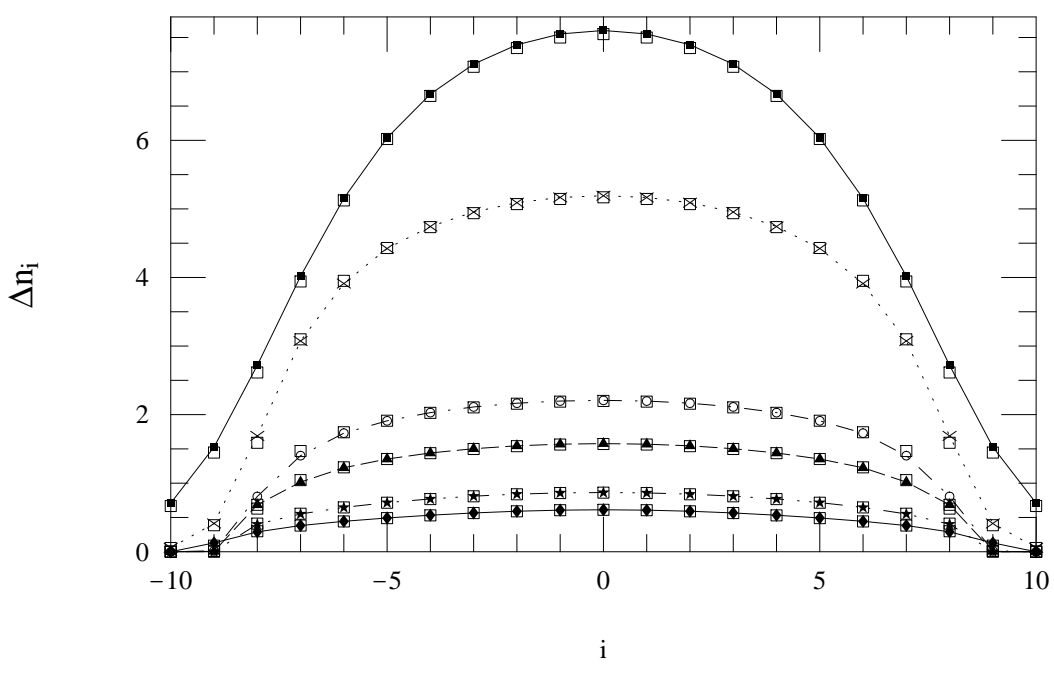

Figure 5: Number fluctuations in the self consistent HFB-Popov approach as a function of lattice site for $V_{\text {eff }}=0.01$ (boxes), $V_{\text {eff }}=0.09$ (crosses), $V_{\text {eff }}=3$ (circles), $V_{e f f}=11$ (triangles), $V_{e f f}=100$ (stars) and $V_{e f f}=312$ (diamonds). The maximum value reached by the profile decreases as $V_{\text {eff }}$ is increased. The empty boxes shown for each of the curves correspond to the number fluctuations predicted by the homogeneous HFB-Popov model using a local density approximation. 


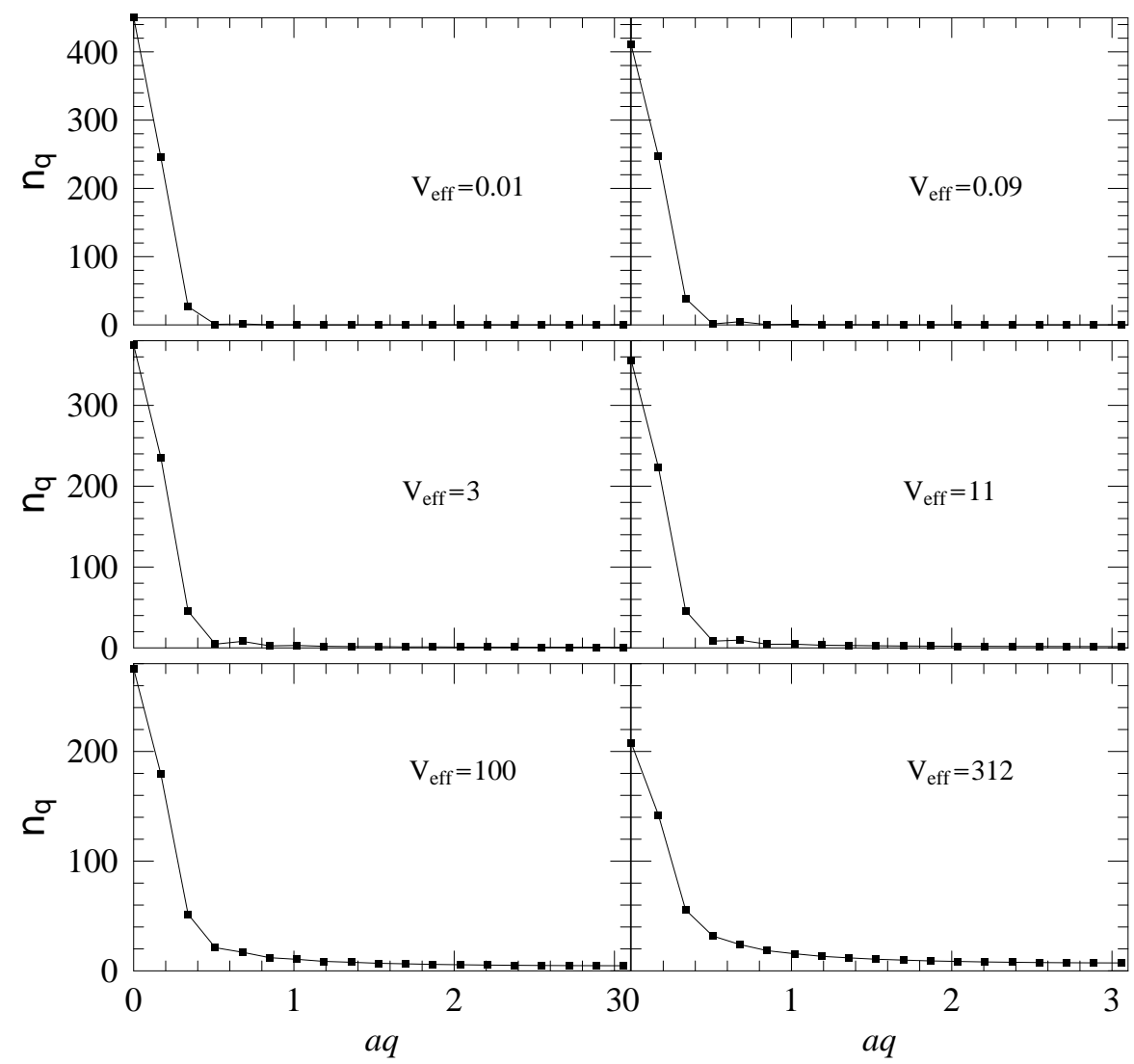

Figure 6: Quasi-momentum distribution as a function of $q a, a$ the lattice spacing, $q$ the quasimomentum, for different values of $V_{\text {eff }}$. 


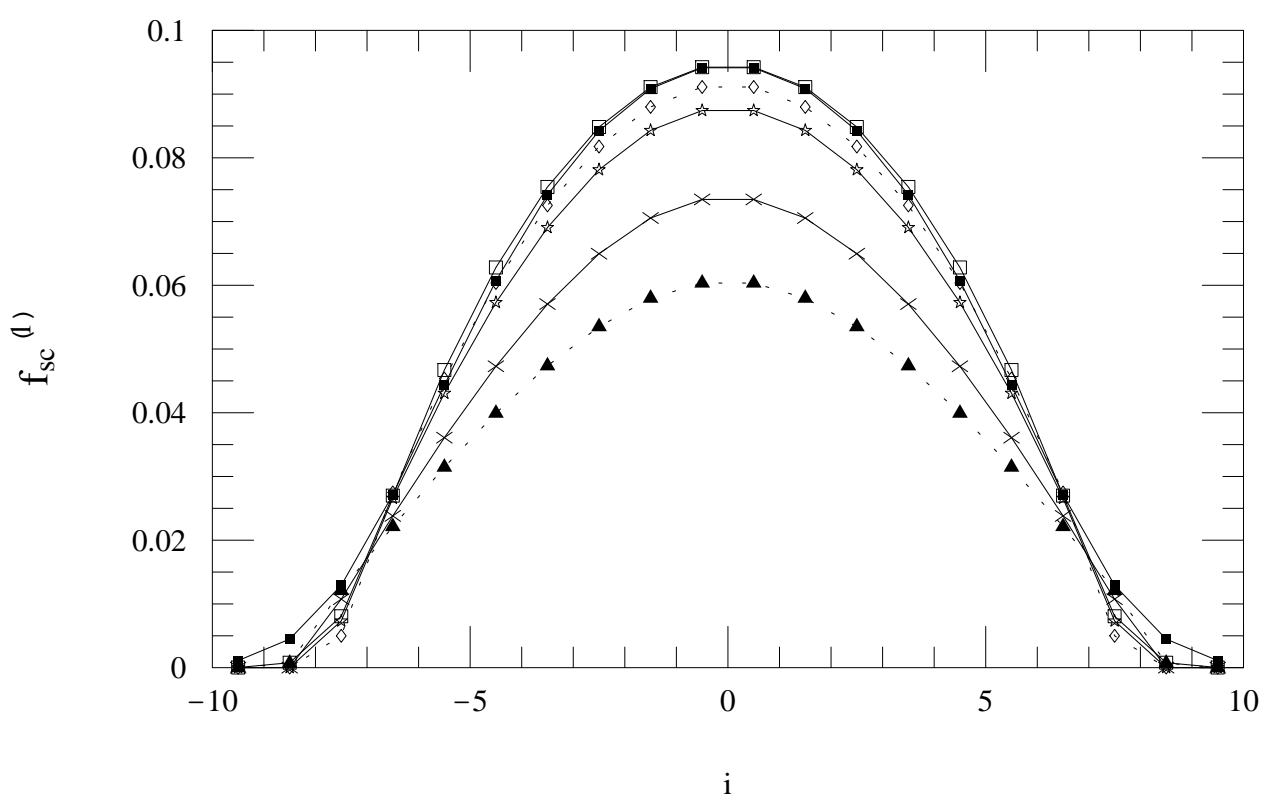

Figure 7: First order on-site superfluid fraction as a function of the lattice site for different values of $V_{\text {eff }}$. Filled boxes: $V_{\text {eff }} .=0.01$, empty boxes: $V_{\text {eff. }}=0.09$, empty diamonds: $V_{\text {eff. }}=3$, stars: $V_{\text {eff. }}=11$, crosses: $V_{\text {eff. }}=100$ and triangles: $V_{\text {eff. }}=312$. 

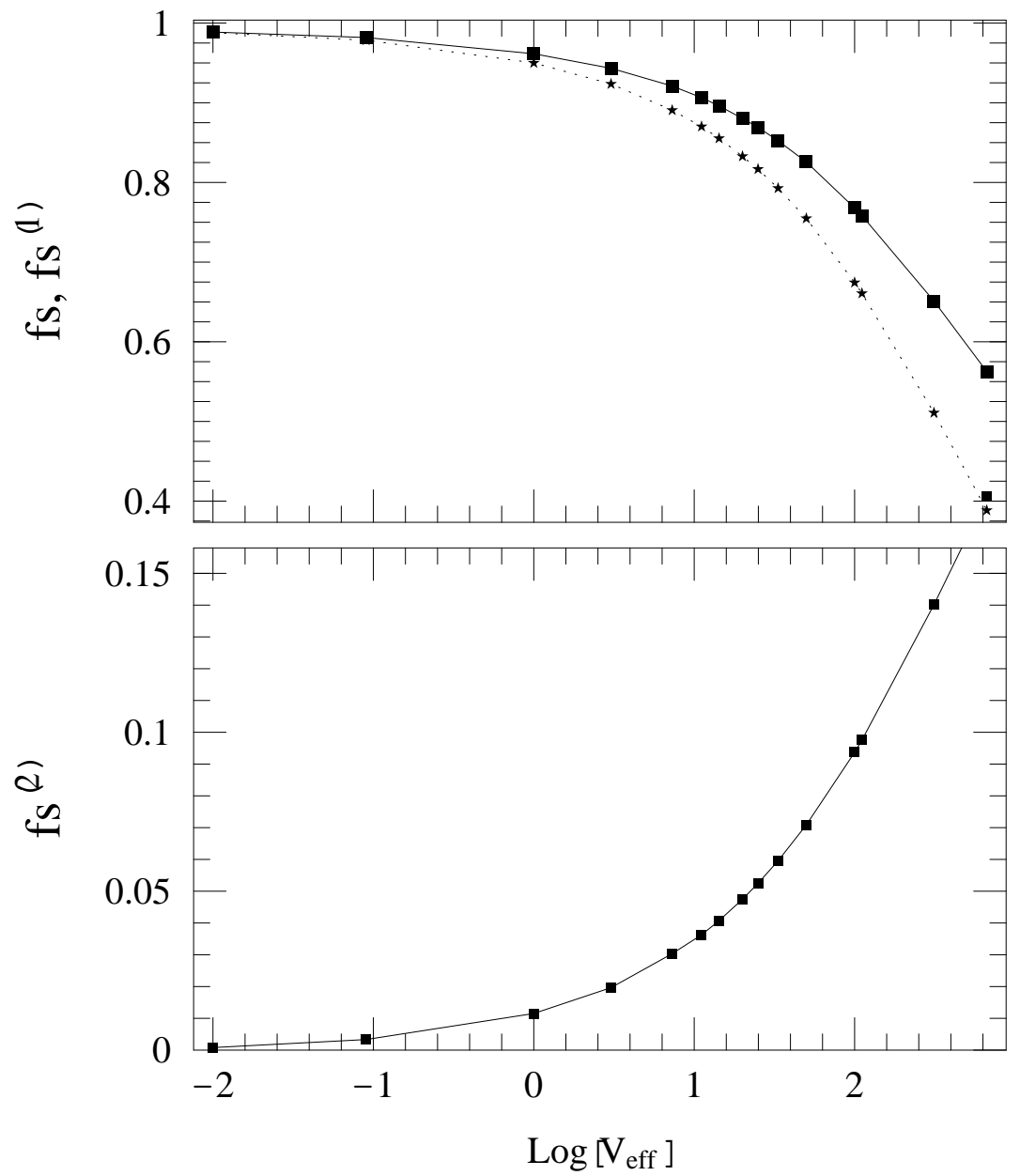

Figure 8: Top panel: First order (boxes) and total (stars) superfluid fraction as a function of $V_{\text {eff. }}$. Bottom panel : second order superfluid fraction as a function of $V_{e f f}$. All these quantities are calculated in the self consistent HFBPopov approach. 